The Free Internet Journal

for Organic Chemistry
Review

Arkivoc 2019, part i, 256-292

\title{
Nucleophilic rhodanine, thiazolidine-2,4-dione and thiazol-4(5H)-one substrates in asymmetric reactions
}

\author{
Sushovan Paladhi, ${ }^{* a}$ Barnali Jana, ${ }^{* b}$ Sudipta Pathak, ${ }^{b}$ and Saikat Kumar Manna ${ }^{b}$ \\ ${ }^{a}$ Department of Chemistry, Anugrah Narayan Singh College, Barh, Patna, Bihar, 803 213, India \\ ${ }^{b}$ Department of Chemistry, Haldia Govt. College, Haldia, Purba Medinipur, West Bengal, 721 657, India \\ Email: ocpaladhi@gmail.com barnalijana09@gmail.com
}

Dedicated to Professor Choong Eui Song on the occasion of his $65^{\text {th }}$ birthday

Received 02-12-2019

Accepted 05-05-2019

Published on line $06-16-2019$

\section{Abstract}

Substituted rhodanines, thiazolidine-2,4-diones and thiazol-4(5H)-ones as nucleophilic substrates have attracted great attention in asymmetric reactions in the past six years due to their high impact in medicinal and pharmaceutical importance. The current review provides a cursory overview of the synthetic methods developed to access the rhodanine, thiazolidine-2,4-dione and thiazol-4(5H)-one substrates and a summary of their applications in catalytic asymmetric reactions to synthesize a broad range of differently substituted chiral derivatives of the corresponding substrates with tertiary/quaternary stereogenic centres.

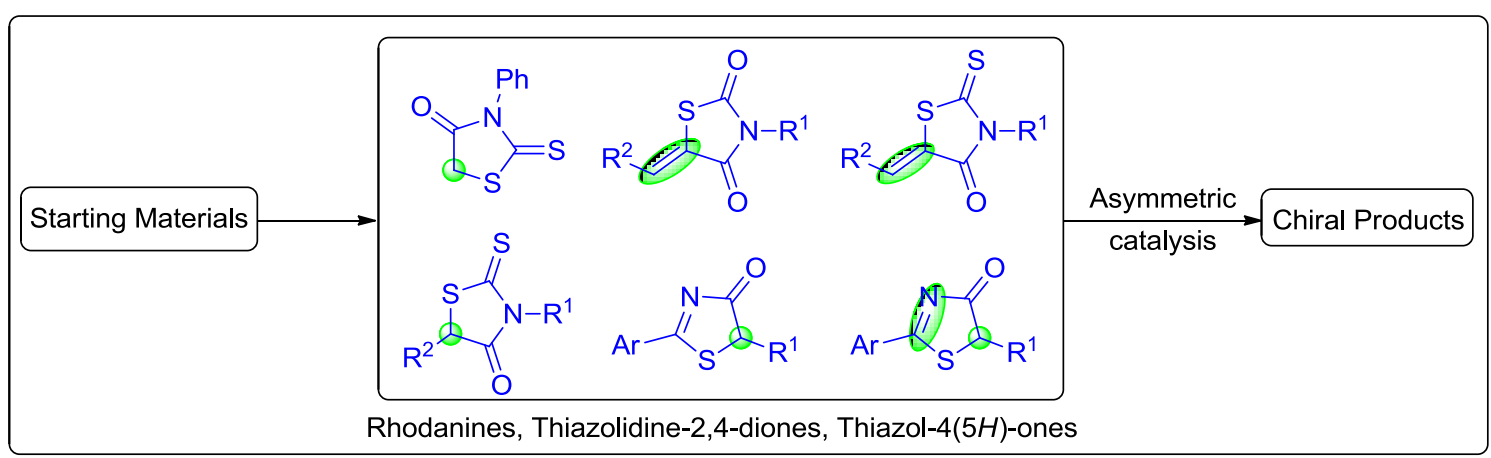

Keywords: Rhodanines, thiazolidine-2,4-diones; thiazol-4(5H)-ones, asymmetric catalysis, metal catalysis, organocatalysis 


\section{Table of Contents}

1. Introduction

2. Synthesis of the Nucleophiles

2.1 Synthesis of $\mathrm{N}$-substituted rhodanines 1

2.2 Synthesis of 5-alkylidene thiazolidine-2,4-diones 2

2.3 Synthesis of 5-alkylidenerhodanines $\mathbf{3}$

2.4 Synthesis of 5-substituted rhodanines 4

2.5 Synthesis of thiazol-4(5H)-ones 5

3. Asymmetric Reactions of Rhodanines, Thiazolidine-2,4-diones and thiazol-4(5H)-ones

3.1 Asymmetric reactions of $N$-phenyl rhodanine (1a)

3.2 Asymmetric reactions of 5-alkylidene thiazolidine-2,4-diones 2

3.3 Asymmetric reactions of 5-alkylidene rhodanines $\mathbf{3}$

3.4 Asymmetric reactions of 5-substituted rhodanines 4

3.5 Asymmetric reactions of thiazol-4(5H)-ones 5

4. Conclusions

\section{Introduction}

Sulfur- and nitrogen-containing five- membered heterocyclic compounds such as rhodanines, thiazolidine-2,4diones and thiazol-4(5H)-ones have become interesting target structures for drug development and discovery. Several compounds of these classes are in clinical trials for diabetic complications, type II diabetes mellitus, antibacterial, antiviral, antimalarial, antifungal, antitumour activities and inhibitors of pancreatic cholesterol esterase. $^{1-21}$ In particular, the rhodanine derivative epalrestat and the thiazolidine-2,4-dione derivatives rosiglitazone, troglitazone and pioglitazone are commercialized for the treatment of diabetes mellitus (Figure 1). ${ }^{22-25}$ Rhodadynes (condensation product of rhodanine and carbonyl compounds), which inhibit the BCL-2 protein, are presently used in cancer therapy. ${ }^{26}$ Rhodanines were also introduced into organic molecules to apply in the development of dye-sensitized solar cells / organic photovoltaic cells, which mainly promoted the absorption spectrum towards red-shift with high efficiency. ${ }^{27-31}$

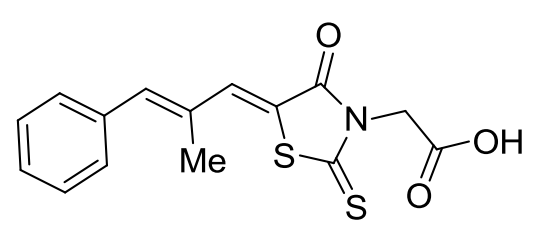

Epalrestat<smiles>[R]COc1ccc(CC2SC(=O)NC2=O)cc1</smiles>

Glitazone<smiles>[R]=[Fe]</smiles><smiles>Cc1c(C)c2c(c(C)c1O)CCC(C)(C)O2</smiles>

Troglitazone

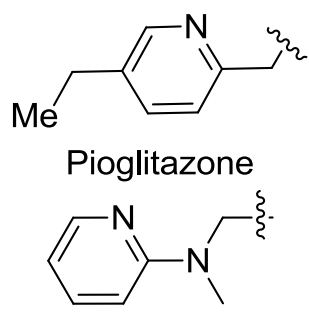

Rosiglitazone

Figure 1. Commercialized drugs from the class of rhodanine and thiazolidine-2,4-dione.

In view of the importance of the rhodanines, thiazolidine-2,4-diones, thiazol-4(5H)-ones and their stereoisomers, in particular of their biological and pharmacological activities, several methods have been established for their facile synthesis. In this review, we summarize the development of enantioselective catalytic methods using rhodanine, thiazolidine-2,4-dione and thiazol-4(5H)-one as nucleophiles to synthesize 
a wide range of their chiral derivatives. A systematic study of the published literature was organised, summarizing the nucleophiles, the synthetic procedures for accessing them, and their application under different asymmetric catalytic methods.

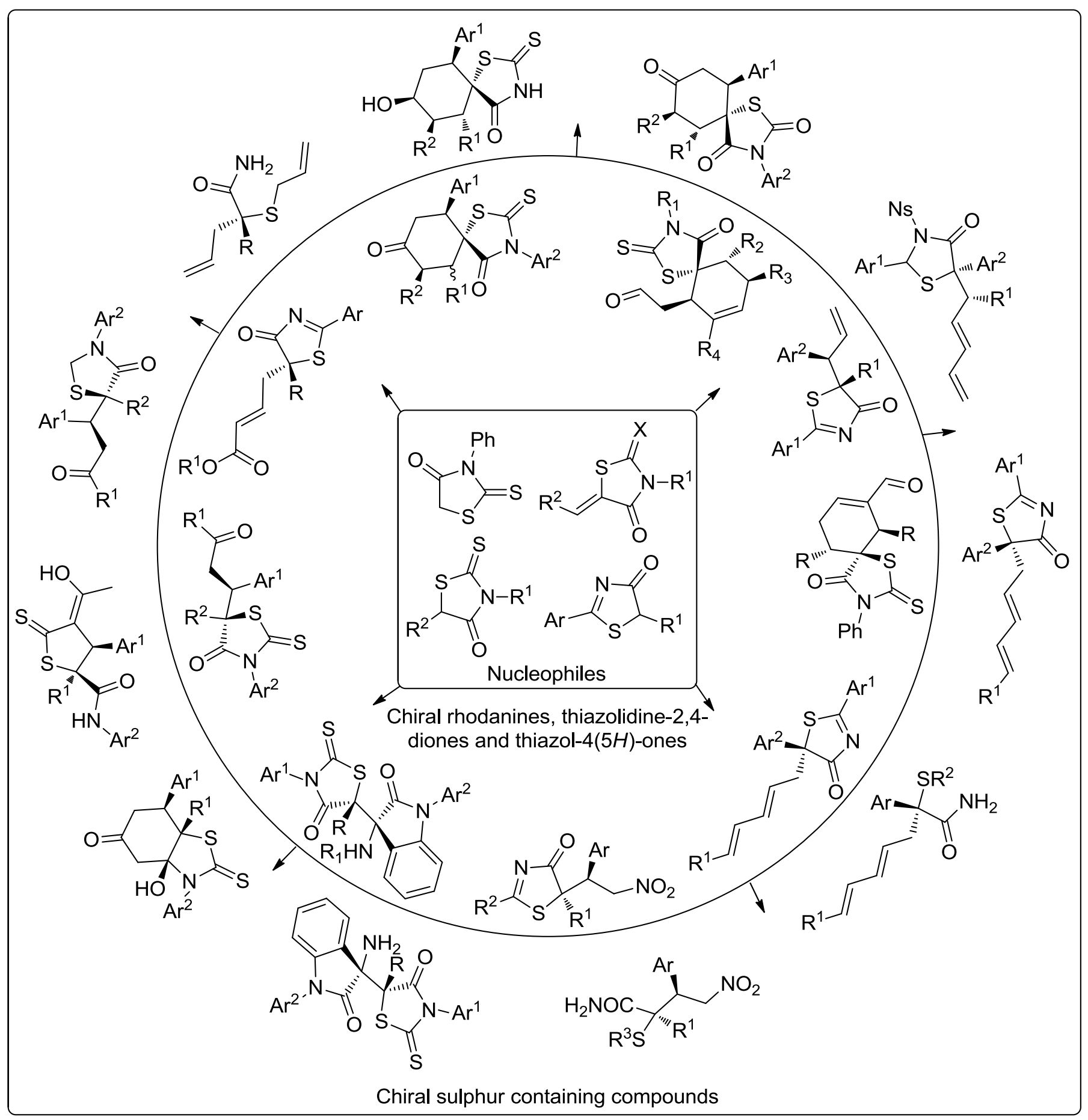

Figure 2. Versatile chiral rhodanines, thiazolidine-2,4-diones and thiazol-4(5H)-ones derivatives and diverse attractive chiral sulphur containing molecules derived from them. 


\section{Synthesis of the Nucleophiles}

The nucleophiles used in the reported literatures are classified into the following five categories (Figure 3):

1. $\mathbf{N}$-Substituted rhodanine substrates $\mathbf{1}$

2. 5-Alkylidenethiazolidine-2,4-dione substrates $\mathbf{2}$

3. 5-Alkylidenerhodanine substrates $\mathbf{3}$

4. 5-Substituted rhodanine substrates 4

5. Thiazol-4(5H)-one substrates 5<smiles>O=C1CSC(=S)N1c1ccccc1</smiles>

1a $N$-Phenylrhodanine<smiles>[R]C=C1SC(=O)N([R])C1=O</smiles>

2 5-Alkylidene thiazolidine-2,4-diones<smiles>[R]C=C1SC(=S)N([R])C1=O</smiles>

3

5-Alkylidene rhodanines<smiles>[R]C1SC(=S)N([R])C1=O</smiles>

4

5-Substituted rhodanines

5

Thiazol-4(5H)-ones

Figure 3. Varieties of rhodanines, thiazolidine-2,4-diones and thiazol-4(5H)-ones used as nucleophiles.

Rhodanine, thiazolidine-2,4-dione and their derivatives are synthesized by various well-known methods. ${ }^{32-35}$ Rhodanine and thiazolidine-2,4-diones are commercially available and can easily be converted into their $\mathrm{N}$-substituted derivatives through simple transformations. ${ }^{36-44}$ However in this review, the methods employed for the preparation of these compounds as starting materials for further asymmetric reactions, are summarized in the sections below.

\subsection{Synthesis of $\boldsymbol{N}$ - substituted rhodanines 1}

Harada et al. reported the direct synthesis of $N$-substituted rhodanines by the reaction of aliphatic/aromatic amines 8 with a stirred solution of bis(carboxymethyl)trithiocarbonate (6) and 1,1'-carbonyldiimidazole (7) in THF (Scheme 1). ${ }^{45}$<smiles>O=C(O)CSC(=S)SCC(=O)O</smiles>

6<smiles>O=C(n1ccnc1)n1ccnc1</smiles>

7

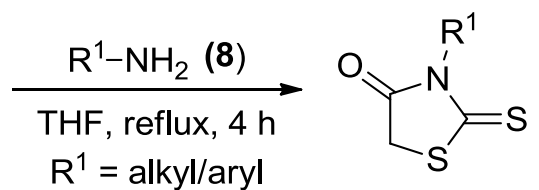

1

Scheme 1. $\mathbf{N}$-Substituted rhodanines 1 from amine 8 . 


\subsection{Synthesis of 5-alkylidenethiazolidine-2,4-diones 2}

5-Alkylidenethiazolidine-2,4-dione 2 can easily be accessed through the synthesis of $N$-substituted thiazolidine-2,4-diones 11 . Initially, the sodium salts of $O$-methyl- $N$-substituted iminothiocarbonates 10 are prepared by the reaction of isothiocyanates 9 with sodium methoxide in dry ether, a method developed by Kristian and co-workers. The obtained products $\mathbf{1 0}$ were treated with bromoacetyl bromide in dichloromethane to obtain $N$-substituted thiazolidine-2,4-diones 11 through the intermediate I (Scheme 2). ${ }^{46}$

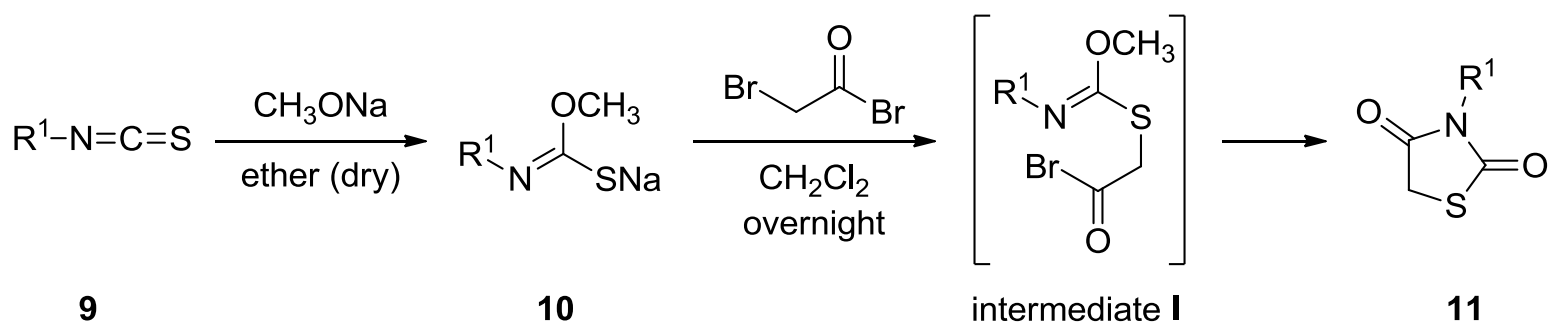

Scheme 2. N-Substituted thiazolidine-2,4-diones 11 from isothiocyanates 9.

Deng et al. $^{47}$ performed the Knoevenagel-condensation of $N$-substituted thiazolidine-2,4-diones 11 with the aldehydes $\mathbf{1 2}$ in the presence of ammonium acetate or $\beta$-alanine in acetic acid to afford the desired 5alkylidenethiazolidine-2,4-diones 2 following the procedure for the synthesis of rhodanine derivatives developed by Harada et al. (Scheme 3 ). ${ }^{45}$

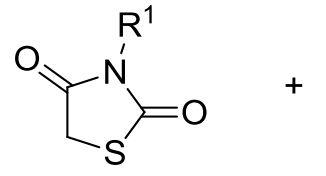

11<smiles>[R]C=O</smiles>

12

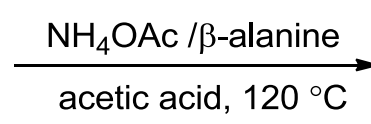<smiles>[R]C=C1SC(=O)N([R])C1=O</smiles>

2

Scheme 3. 5-Alkylidenethiazolidine-2,4-diones 2 from $\mathbf{N}$-substituted thiazolidine-2,4-diones 11

\subsection{Synthesis of 5 -alkylidenerhodanines 3}

5-Alkylidenerhodanines 3 have been prepared by Knoevenagel condensations of $N$-substituted rhodanines 1 with aldehydes $\mathbf{1 2}$ in acetic acid in the presence of ammonium acetate or $\beta$-alanine as reported by Harada et al. (Scheme 4). ${ }^{45}$<smiles>[R]N1C(=O)CSC1=S</smiles>

1

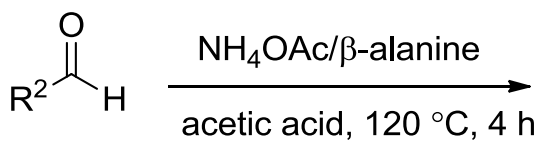

12<smiles>[R]C=C1SC(=S)N([R])C1=O</smiles>

3

Scheme 4. 5-Alkylidenerhodanines 3 from $N$ - substituted rhodanines 1

Alternatively, 5-alkylidenerhodanines 3 can be prepared through two-step reactions (Scheme 5). First, intermediates $\mathbf{1 4}$ were synthesized according to the method developed by Yurttaş et al. by the reaction of dry 
ammonium aryldithiocarbamates 13 with oxalyl chloride in anhydrous benzene. The intermediates 14 were then subjected to Wittig reaction conditions in anhydrous toluene to obtain desired 5-alkylidenerhodanines $3^{48}$

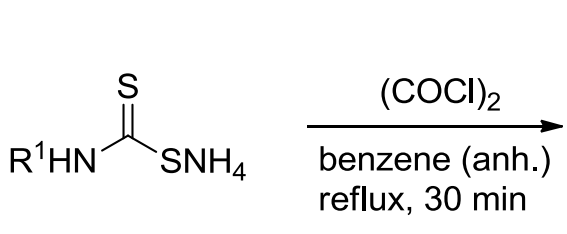

13

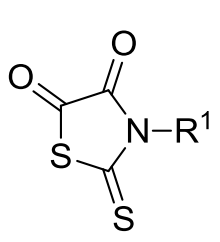

14

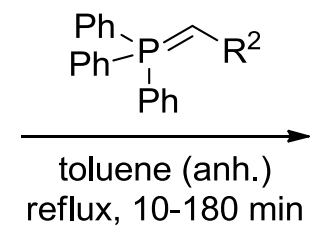

toluene $($ anh.)
reflux, $10-180 \mathrm{~min}$<smiles>[R]C=C1SC(=S)N([R])C1=O</smiles>

3

Scheme 5. 5-Alkylidenerhodanines 3 from ammonium $N$-aryldithiocarbamates 13

\subsection{Synthesis of 5-substituted rhodanines 4}

Ye et al. reported the syntheses of 5 -substituted rhodanines 4 by the reaction of an $\alpha$-mercaptocarboxylic acid 15 with isothiocyanates 9 in 1,4-dioxane in the presence of triethylamine at $0{ }^{\circ} \mathrm{C}($ Scheme 6$) .{ }^{49}$

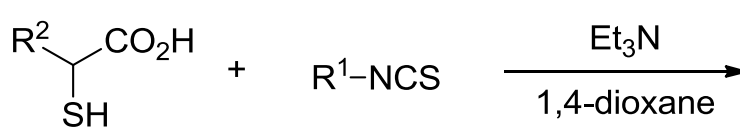

15
9<smiles>[R]C1SC(=S)N([R1])C1=O</smiles>

4

Scheme 6. 5-Substituted rhodanines 4 from $\alpha$-mercaptocarboxylic acids 15.

\subsection{Synthesis of thiazol-4(5H)-ones 5}

Thiazol-4(5H)-ones 5 were prepared through three different pathways, depending on the substituents. ${ }^{50,51}$

2.5.1. Path A. Thiazol-4(5H)-ones can be synthesized by treating $\alpha$-mercaptocarboxylic acids 16 with carbonitriles $\mathbf{1 7}$ in the presence of either catalytic amount of pyridine or in the presence of triethylamine in ethanol under reflux condition (Scheme 7).

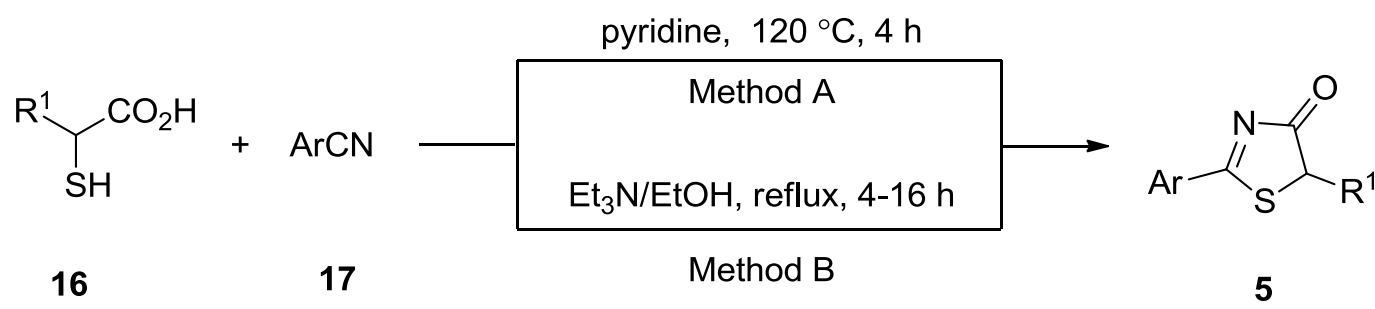

Scheme 7. Thiazol-4(5H)-ones 5 from $\alpha$-mercaptocarboxylic acids 16.

2.5.2. Path B. Alternatively, thiazol-4(5H)-ones $\mathbf{5}$ have been accessed by the coupling of arylthioamide $\mathbf{2 1}$ with ethyl 2-bromoester $\mathbf{1 9}$ or the acid chloride $\mathbf{2 0}$ in pyridine, which were prepared from the corresponding 2-bromoalkanoic acid 18 through well-established methods (Scheme 8). 


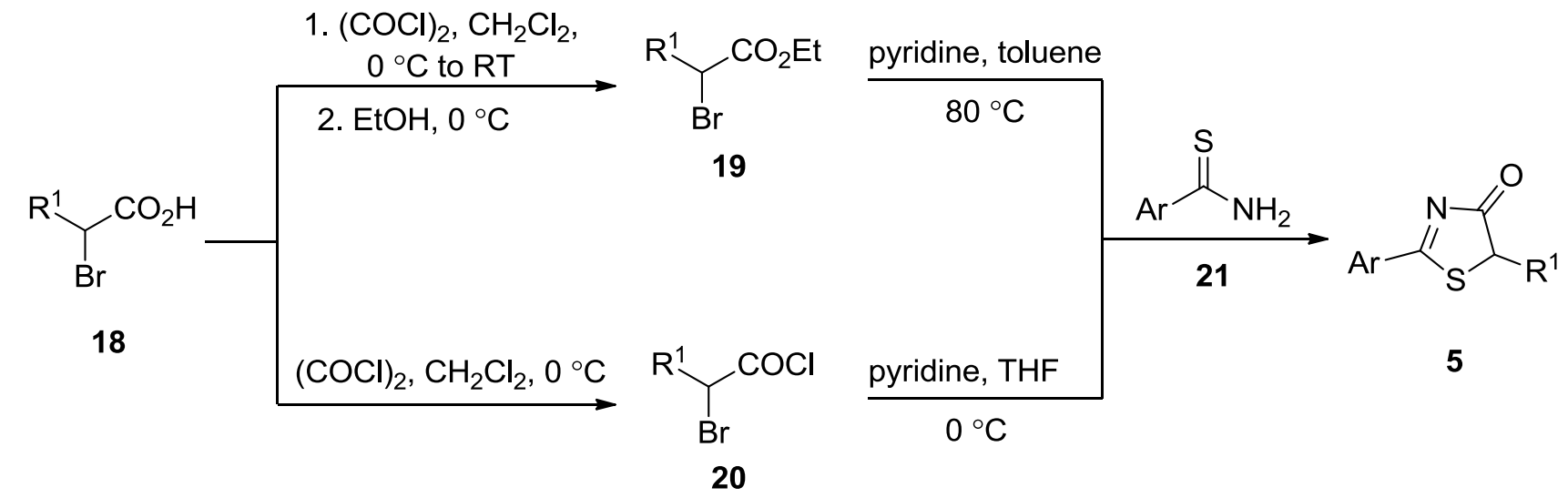

Scheme 8. Thiazol-4(5H)-ones 5 from 2-bromoalkanoic acid 18.

\section{Asymmetric Reactions of Rhodanines, Thiazolidine-2,4-diones, and Thiazol-4(5H)-ones}

\subsection{Asymmetric reactions of $\mathbf{N}$-phenylrhodanine (1a)}

The first examples of an enantioselective organocatalytic Michael/Michael/aldol reaction of enals and the double Michael addition of aromatic dienones with bisnucleophile $N$-phenylrhodanine (1a) were reported in 2013 by Veselý et al. (Schemes 9, 10). ${ }^{52}$

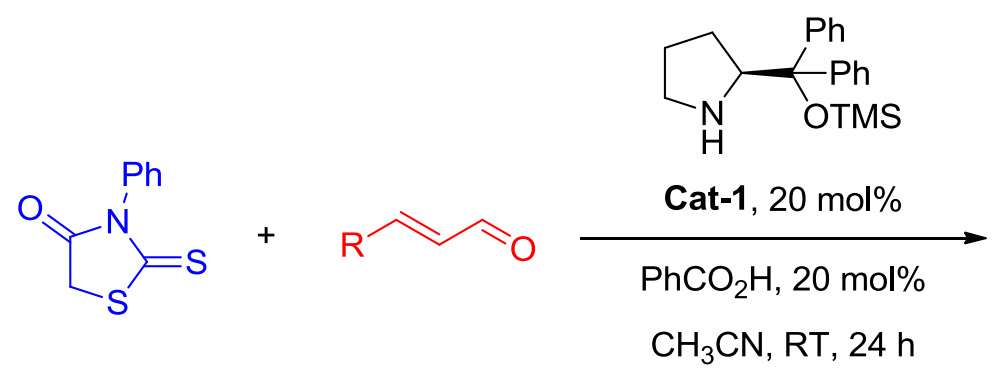

$1 \mathbf{a}$<smiles>CCC1CC=C(C=O)C(CC)[C@@]12SC(=S)N(c1ccccc1)C2=O</smiles>

23a, yield $63 \%$ dr $20: 1$, ee $99 \%$

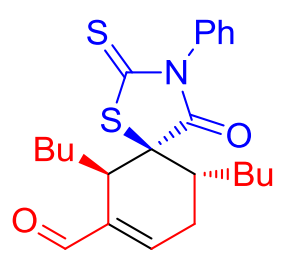

23b, yield $61 \%$ dr 5:1, ee $99 \%$

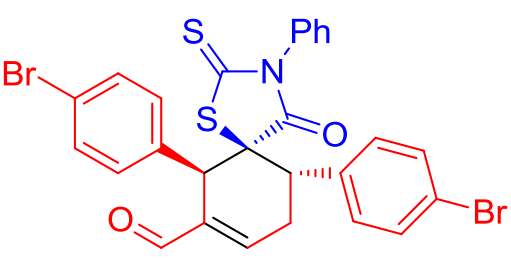

23c, yield $50 \%$ dr $16: 1$, ee $99 \%$<smiles>[R]C1CC=C(C=O)C([R])[C@]12SC(=S)N(c1ccccc1)C2=O</smiles>

23<smiles>O=CC1=CC[C@H](c2ccccc2)[C@]2(SC(=S)N(c3ccccc3)C2=O)[C@H]1c1ccccc1</smiles>

23d, yield $50 \%$

dr $7: 1$, ee $99 \%$

Scheme 9. Michael Michael/Michael/Aldol reaction of $N$-phenylrhodanine (1a) with enals 22

The Michael/Michael/aldol reaction of enals 22 with bisnucleophile $N$-phenylrhodanine (1a) occurs in presence of a proline-based catalyst Cat-1 with good yields (up to 63\%) and excellent stereoselectivities (dr up to $20: 1$ and ee up to $99 \%)$. 
Furthermore, the double Michael addition between trans,trans-dibenzylideneacetone (24) and $\mathrm{N}$-phenylrhodanine (1a) occurs in the presence of a cinchona-based primary-amine catalyst Cat- $\mathbf{2}$ in combination with the additive 2,4-dinitrobenzoic acid (DNBA) in acetonitrile to afford the best result in respect to yield (83\%) and stereoselectivity (dr 20:1 and ee 75\%).

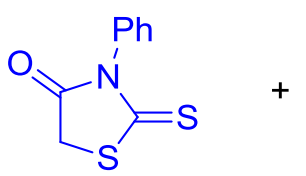

$1 \mathbf{a}$

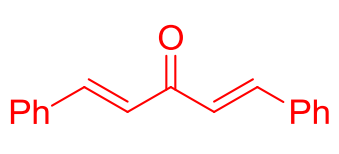

24

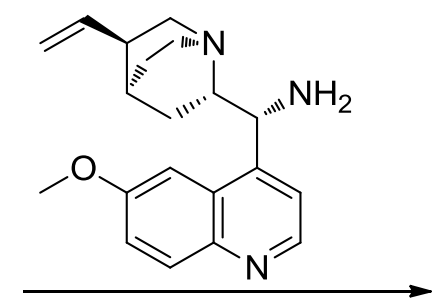

Cat-2, 20 mol\% DNBA (20 mol\%) $\mathrm{CH}_{3} \mathrm{CN}, \mathrm{RT}, 96 \mathrm{~h}$<smiles>O=C1C[C@H](c2ccccc2)[C@@]2(SC1=S)C(=O)N(c1ccccc1)C2c1ccccc1</smiles>

25 , yield $83 \%$ dr $20: 1$, ee $75 \%$

Scheme 10. Michael addition of $N$-phenylrhodanine (1a) with trans, trans-dibenzylideneacetone (24).

\subsection{Asymmetric reactions of 5-alkylidenethiazolidine-2,4-diones 2}

In 2015, Deng and co-workers reported the asymmetric 1,3-dipolar cycloaddition of 5-alkylidenethiazolidine2,4-diones 2 with azomethine ylides 26 using chiral N,O-ligand 27 in combination with $\mathrm{Cu}\left(\mathrm{CH}_{3} \mathrm{CN}_{4} \mathrm{BF}_{4}(\mathrm{Scheme}\right.$ 11, Table 1). ${ }^{47}$ The exo-spirocyclic pyrrolidine-thia(oxa)zolidinediones products 28 were obtained in high yields (up to 99\%) with excellent levels of stereoselectivity (dr up to 99:1; ee up to 98\%).<smiles>[R]C=C1SC(=O)N([R])C1=O</smiles>

2<smiles>[R]/C=N\CC(C)=O</smiles>

26<smiles>C[C@H]1[C@H](C(O)(c2ccccc2)c2ccccc2)N=C2C=Cc3cc(C(F)(F)F)ccc3N21</smiles>

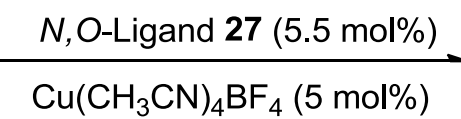
$\mathrm{Et}_{3} \mathrm{~N}(20 \mathrm{~mol} \%)$

$\mathrm{CH}_{2} \mathrm{Cl}_{2}, 0^{\circ} \mathrm{C}$<smiles>[R]C1N[C@@H](C(C)=O)C([R])[C@@]12SC(=O)N([R])C2=O</smiles>

28

Scheme 11. Asymmetric 1,3-dipolar cycloaddition of 5-alkylidenethiazolidine-2,4-diones 2 with azomethine ylides 26. 
Table 1

\begin{tabular}{|c|c|c|c|c|c|c|}
\hline Entry & $\mathrm{R}^{1}$ & $\mathrm{R}^{2}$ & $\mathrm{R}^{3}$ & Yield (\%) & $\mathrm{dr}$ & ee $(\%)$ \\
\hline 1 & $\mathrm{Ph}$ & $\mathrm{Ph}$ & $p-\mathrm{ClC}_{6} \mathrm{H}_{4}$ & 95 & $97: 3$ & 96 \\
\hline 2 & $\mathrm{Ph}$ & $\mathrm{Ph}$ & $\mathrm{Ph}$ & 98 & $97: 3$ & 96 \\
\hline 3 & $\mathrm{Ph}$ & $\mathrm{Ph}$ & $o-\mathrm{ClC}_{6} \mathrm{H}_{4}$ & 80 & $87: 13$ & 90 \\
\hline 4 & $\mathrm{Ph}$ & $\mathrm{Ph}$ & $m-\mathrm{ClC}_{6} \mathrm{H}_{4}$ & 98 & $97: 3$ & 96 \\
\hline 5 & $\mathrm{Ph}$ & $\mathrm{Ph}$ & $p-\mathrm{BrC}_{6} \mathrm{H}_{4}$ & 98 & $97: 3$ & 97 \\
\hline 6 & $\mathrm{Ph}$ & $\mathrm{Ph}$ & $p-\mathrm{CF}_{3} \mathrm{C}_{6} \mathrm{H}_{4}$ & 99 & $98: 2$ & 96 \\
\hline 7 & $\mathrm{Ph}$ & $\mathrm{Ph}$ & $o-\mathrm{MeC}_{6} \mathrm{H}_{4}$ & 95 & $86: 14$ & 89 \\
\hline 8 & $\mathrm{Ph}$ & $\mathrm{Ph}$ & $p-\mathrm{MeC}_{6} \mathrm{H}_{4}$ & 98 & $97: 3$ & 96 \\
\hline 9 & $\mathrm{Ph}$ & $\mathrm{Ph}$ & $p-\mathrm{MeOC}_{6} \mathrm{H}_{4}$ & 98 & $97: 3$ & 95 \\
\hline 10 & $\mathrm{Ph}$ & $\mathrm{Ph}$ & 2-naphthyl & 99 & $97: 3$ & 96 \\
\hline 11 & $\mathrm{Ph}$ & $\mathrm{Ph}$ & 2-furyl & 93 & $94: 6$ & 93 \\
\hline 12 & $\mathrm{Ph}$ & $\mathrm{Ph}$ & ${ }^{i} \mathrm{Bu}$ & 90 & $88: 12$ & 87 \\
\hline 13 & $\mathrm{Ph}$ & $p-\mathrm{ClC}_{6} \mathrm{H}_{4}$ & $p-\mathrm{ClC}_{6} \mathrm{H}_{4}$ & 98 & $97: 3$ & 95 \\
\hline 14 & $\mathrm{Ph}$ & $m-\mathrm{ClC}_{6} \mathrm{H}_{4}$ & $p-\mathrm{ClC}_{6} \mathrm{H}_{4}$ & 95 & $98: 2$ & 96 \\
\hline 15 & $\mathrm{Ph}$ & $p-\mathrm{MeOC}_{6} \mathrm{H}_{4}$ & $p-\mathrm{ClC}_{6} \mathrm{H}_{4}$ & 90 & 99:1 & 96 \\
\hline 16 & $\mathrm{Ph}$ & $2,6-\mathrm{Me}_{2} \mathrm{C}_{6} \mathrm{H}_{3}$ & $p-\mathrm{ClC}_{6} \mathrm{H}_{4}$ & 40 & $88: 12$ & 95 \\
\hline 17 & $p-\mathrm{BrC}_{6} \mathrm{H}_{4}$ & $\mathrm{Ph}$ & $p-\mathrm{ClC}_{6} \mathrm{H}_{4}$ & 98 & $98: 2$ & 98 \\
\hline 18 & $m-\mathrm{BrC}_{6} \mathrm{H}_{4}$ & $\mathrm{Ph}$ & $p-\mathrm{ClC}_{6} \mathrm{H}_{4}$ & 99 & $97: 3$ & 94 \\
\hline 19 & $o-\mathrm{BrC}_{6} \mathrm{H}_{4}$ & $\mathrm{Ph}$ & $p-\mathrm{ClC}_{6} \mathrm{H}_{4}$ & 98 & $98: 2$ & 95 \\
\hline 20 & $p-\mathrm{O}_{2} \mathrm{NC}_{6} \mathrm{H}_{4}$ & $\mathrm{Ph}$ & $p-\mathrm{ClC}_{6} \mathrm{H}_{4}$ & 99 & $97: 3$ & 97 \\
\hline 21 & $p-\mathrm{MeOC}_{6} \mathrm{H}_{4}$ & $\mathrm{Ph}$ & $p-\mathrm{ClC}_{6} \mathrm{H}_{4}$ & 95 & $98: 2$ & 97 \\
\hline 22 & $m-\mathrm{MeOC}_{6} \mathrm{H}_{4}$ & $\mathrm{Ph}$ & $p-\mathrm{ClC}_{6} \mathrm{H}_{4}$ & 98 & $98: 2$ & 96 \\
\hline 23 & 2-furyl & $\mathrm{Ph}$ & $p-\mathrm{ClC}_{6} \mathrm{H}_{4}$ & 93 & $96: 4$ & 94 \\
\hline 24 & $\mathrm{PhCH}=\mathrm{CH}$ & $\mathrm{Ph}$ & $p-\mathrm{ClC}_{6} \mathrm{H}_{4}$ & 85 & $96: 4$ & 94 \\
\hline 25 & $\mathrm{CO}_{2} \mathrm{Et}$ & $\mathrm{Ph}$ & $p-\mathrm{ClC}_{6} \mathrm{H}_{4}$ & 85 & $80: 20$ & 89 \\
\hline 26 & $\mathrm{H}$ & $\mathrm{Ph}$ & $p-\mathrm{ClC}_{6} \mathrm{H}_{4}$ & 80 & $86: 14$ & 94 \\
\hline 27 & ${ }^{n} \operatorname{Pr}$ & $\mathrm{Ph}$ & $p-\mathrm{ClC}_{6} \mathrm{H}_{4}$ & 80 & $97: 3$ & 95 \\
\hline 28 & $\mathrm{Ph}$ & Me & $p-\mathrm{ClC}_{6} \mathrm{H}_{4}$ & 98 & $98: 2$ & 94 \\
\hline
\end{tabular}

\subsection{Asymmetric reactions of $\mathbf{5}$-alkylidenerhodanines $\mathbf{3}$}

The development of asymmetric reaction of 5-alkylidenerhodanines is known since 2012. The first report by Ye and co-workers was the asymmetric cascade reaction between acyclic $\alpha, \beta$-unsaturated ketones 29 and 5 alkylidenerhodanines 3 . $^{53}$ 
<smiles>[R]C=C1SC(=S)N([R1])C1=O</smiles>

3

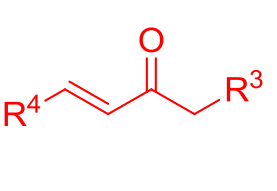

29

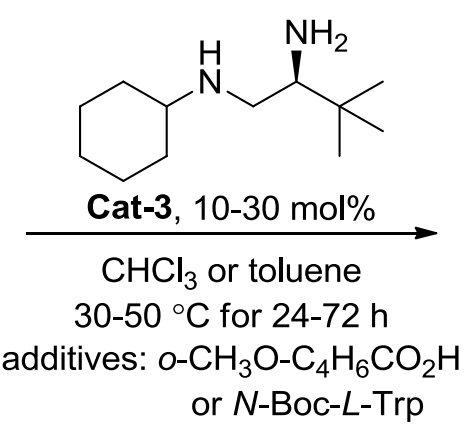

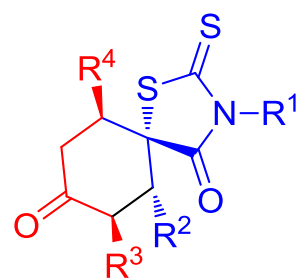

30

Scheme 12. Asymmetric cascade reaction of 5-alkylidenerhodanines 3 with $\alpha, \beta$-unsaturated ketones 29.

Table 2

\begin{tabular}{|c|c|c|c|c|c|c|c|}
\hline Entry & $\mathrm{R}^{1}$ & $\mathrm{R}^{2}$ & $\mathrm{R}^{3}$ & $\mathrm{R}^{4}$ & Yield (\%) & $\mathrm{dr}$ & ee $(\%)$ \\
\hline 1 & $\mathrm{Ph}$ & $\mathrm{CO}_{2} \mathrm{Et}$ & $\mathrm{H}$ & $\mathrm{Ph}$ & 86 & $>20: 1$ & 98 \\
\hline 2 & $\mathrm{Ph}$ & $\mathrm{CO}_{2} \mathrm{Et}$ & $\mathrm{H}$ & $o-\mathrm{FC}_{6} \mathrm{H}_{4}$ & 83 & $13: 1$ & 92 \\
\hline 3 & $\mathrm{Ph}$ & $\mathrm{CO}_{2} \mathrm{Et}$ & $\mathrm{H}$ & $p-\mathrm{BrC}_{6} \mathrm{H}_{4}$ & 81 & $>20: 1$ & 87 \\
\hline 4 & $\mathrm{Ph}$ & $\mathrm{CO}_{2} \mathrm{Et}$ & $\mathrm{H}$ & $o-\mathrm{MeOC}_{6} \mathrm{H}_{4}$ & 79 & $9: 1$ & 88 \\
\hline 5 & $\mathrm{Ph}$ & $\mathrm{CO}_{2} \mathrm{Et}$ & $\mathrm{H}$ & $m-\mathrm{MeC}_{6} \mathrm{H}_{4}$ & 80 & $>20: 1$ & 98 \\
\hline 6 & $\mathrm{Ph}$ & $\mathrm{CO}_{2} \mathrm{Et}$ & $\mathrm{H}$ & $p-\mathrm{MeC}_{6} \mathrm{H}_{4}$ & 80 & $>20: 1$ & $>99$ \\
\hline 7 & $\mathrm{Ph}$ & $\mathrm{CO}_{2} \mathrm{Et}$ & $\mathrm{H}$ & 2-thienyl & 90 & $18: 1$ & 90 \\
\hline 8 & $\mathrm{Ph}$ & $\mathrm{CO}_{2} \mathrm{Et}$ & $\mathrm{H}$ & $\mathrm{CO}_{2} \mathrm{Et}$ & 35 & $>20: 1$ & 94 \\
\hline 9 & $\mathrm{Ph}$ & $\mathrm{CO}_{2} \mathrm{Et}$ & \multicolumn{2}{|c|}{ cyclohexenone (29) } & 95 & $>20: 1$ & 0 \\
\hline 10 & $\mathrm{Ph}$ & $\mathrm{Ph}$ & $\mathrm{H}$ & $\mathrm{Ph}$ & 70 & $>20: 1$ & 94 \\
\hline 11 & $\mathrm{Ph}$ & $p-\mathrm{BrC}_{6} \mathrm{H}_{4}$ & $\mathrm{H}$ & $\mathrm{Ph}$ & 94 & $>20: 1$ & 98 \\
\hline 12 & $\mathrm{Ph}$ & $p-\mathrm{FC}_{6} \mathrm{H}_{4}$ & $\mathrm{H}$ & $\mathrm{Ph}$ & 86 & $>20: 1$ & 95 \\
\hline 13 & $\mathrm{Ph}$ & $p-\mathrm{O}_{2} \mathrm{NC}_{6} \mathrm{H}_{4}$ & $\mathrm{H}$ & $\mathrm{Ph}$ & 85 & $>20: 1$ & 98 \\
\hline 14 & $\mathrm{Ph}$ & $p-\mathrm{MeOC}_{6} \mathrm{H}_{4}$ & $\mathrm{H}$ & $\mathrm{Ph}$ & 68 & $>20: 1$ & 99 \\
\hline 15 & $\mathrm{H}$ & $\mathrm{CO}_{2} \mathrm{Et}$ & $\mathrm{H}$ & $\mathrm{Ph}$ & 90 & $9: 1$ & 99 \\
\hline 16 & $\mathrm{H}$ & $\mathrm{CO}_{2} \mathrm{Et}$ & $\mathrm{H}$ & $m-\mathrm{MeC}_{6} \mathrm{H}_{4}$ & 77 & $8: 1$ & 89 \\
\hline 17 & $\mathrm{CH}\left(\mathrm{CH}_{3}\right)_{2}$ & $p-\mathrm{BrC}_{6} \mathrm{H}_{4}$ & $\mathrm{H}$ & $\mathrm{Ph}$ & 88 & $>20: 1$ & 98 \\
\hline 18 & cyclohexyl & $p-\mathrm{BrC}_{6} \mathrm{H}_{4}$ & $\mathrm{H}$ & $\mathrm{Ph}$ & 87 & $>20: 1$ & 99 \\
\hline 19 & $\mathrm{Ph}$ & $\mathrm{CO}_{2} \mathrm{Et}$ & Et & $\mathrm{Ph}$ & 85 & $12: 1$ & 99 \\
\hline 20 & $\mathrm{Ph}$ & $p-\mathrm{BrC}_{6} \mathrm{H}_{4}$ & Et & $\mathrm{Ph}$ & 43 & $8: 1$ & 99 \\
\hline 21 & $\mathrm{Ph}$ & $\mathrm{CO}_{2} \mathrm{Et}$ & Me & $\mathrm{Ph}$ & 87 & $10: 1$ & 98 \\
\hline 22 & $\mathrm{Ph}$ & $p-\mathrm{FC}_{6} \mathrm{H}_{4}$ & Me & $\mathrm{Ph}$ & 83 & $7: 1$ & 93 \\
\hline 23 & $\mathrm{H}$ & $\mathrm{CO}_{2} \mathrm{Et}$ & Me & $\mathrm{Ph}$ & 78 & $8: 1$ & 97 \\
\hline 24 & $\mathrm{H}$ & $\mathrm{CO}_{2} \mathrm{Et}$ & Me & 2-furanyl & 82 & $5: 1$ & 96 \\
\hline
\end{tabular}

The reaction was promoted by a simple chiral diamine catalyst Cat-3 to afford spirocyclohexanonerhodanines $\mathbf{3 0}$ with multiple contiguous chiral centers with high stereoselectivities (up to >99\% ee and >20:1 dr) (Scheme 12, Table 2). However, the enantioselectivity for the cascade reaction dropped to $0 \%$ ee when the reaction was performed in presence of cyclic $\alpha, \beta$-unsaturated ketones (Table 2, entry 9). 
In 2013, the same group further explored diphenylprolinol silyl ether Cat-1-catalyzed asymmetric DielsAlder reaction between 2,4-dienals 31 and 5-alkylidenerhodanines $\mathbf{3}$ derivatives to access various spirocyclic compounds 32 with good yields (up to 98\%) and excellent stereoselectivities (up to 99\% ee and >19: $1 \mathrm{dr}$ ). The reaction proceeded via trienamine intermediate, which is formed by the catalyst and the dienal substrate (Scheme 13, Table 3)..$^{54}$<smiles>[R]C=C1SC(=S)N([R])C1=O</smiles>

3<smiles>[R]C=C([R])C=CC=O</smiles>

31

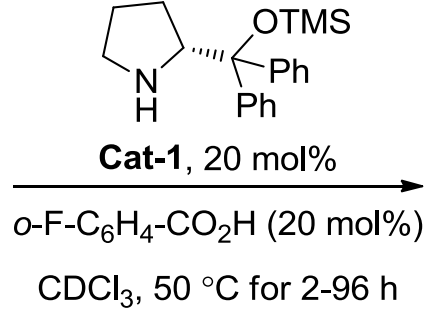

$\mathrm{CDCl}_{3}, 50^{\circ} \mathrm{C}$ for $2-96 \mathrm{~h}$

Scheme 13. Asymmetric Diels-Alder reaction between 5-alkylidenerhodanines 3 and 2,4-dienals 31.

\section{Table 3}

\begin{tabular}{|c|c|c|c|c|c|c|c|}
\hline Entry & $\mathrm{R}^{1}$ & $\mathrm{R}^{2}$ & $\mathrm{R}^{3}$ & $\mathrm{R}^{4}$ & Yield (\%) & $\mathrm{dr}$ & ee (\%) \\
\hline 1 & cyclohexyl & $p-\mathrm{BrC}_{6} \mathrm{H}_{4}$ & $\mathrm{H}$ & $\mathrm{H}$ & 90 & $>19: 1$ & 94 \\
\hline 2 & $\mathrm{Ph}$ & $p-\mathrm{FC}_{6} \mathrm{H}_{4}$ & $\mathrm{H}$ & $\mathrm{H}$ & 82 & $10: 1$ & 93 \\
\hline 3 & $\mathrm{Ph}$ & $p-\mathrm{O}_{2} \mathrm{NC}_{6} \mathrm{H}_{4}$ & $\mathrm{H}$ & $\mathrm{H}$ & 91 & $>19: 1$ & 90 \\
\hline 4 & $\mathrm{Ph}$ & $\mathrm{Ph}$ & $\mathrm{H}$ & $\mathrm{H}$ & 84 & $>19: 1$ & 92 \\
\hline 5 & $\mathrm{Ph}$ & $p-\mathrm{MeOC}_{6} \mathrm{H}_{4}$ & $\mathrm{H}$ & $\mathrm{H}$ & 64 & $>19: 1$ & 91 \\
\hline 6 & $\mathrm{Ph}$ & $p-\mathrm{O}_{2} \mathrm{NC}_{6} \mathrm{H}_{4}$ & $\mathrm{H}$ & $\mathrm{H}$ & 95 & $>19: 1$ & 92 \\
\hline 7 & $\mathrm{Ph}$ & $\mathrm{CO}_{2} \mathrm{Et}$ & $\mathrm{H}$ & $\mathrm{H}$ & 93 & $>19: 1$ & 94 \\
\hline 8 & $\mathrm{Ph}$ & $p-\mathrm{BrC}_{6} \mathrm{H}_{4}$ & $\mathrm{H}$ & $\mathrm{H}$ & 76 & $>19: 1$ & 93 \\
\hline 9 & cyclohexyl & $\mathrm{Ph}$ & $\mathrm{H}$ & $\mathrm{H}$ & 88 & $>19: 1$ & 94 \\
\hline 10 & ${ }^{i} \mathrm{Pr}$ & $p-\mathrm{BrC}_{6} \mathrm{H}_{4}$ & $\mathrm{H}$ & $\mathrm{H}$ & 89 & $>19: 1$ & 93 \\
\hline 11 & $\mathrm{Ph}$ & $p-\mathrm{FC}_{6} \mathrm{H}_{4}$ & $\mathrm{Me}$ & $\mathrm{Me}$ & 98 & $10: 1$ & 96 \\
\hline 12 & $\mathrm{Ph}$ & $\mathrm{CO}_{2} \mathrm{Et}$ & $\mathrm{Me}$ & $\mathrm{Me}$ & 91 & $>19: 1$ & 96 \\
\hline 13 & cyclohexyl & $\mathrm{Ph}$ & $\mathrm{Me}$ & $\mathrm{Me}$ & 94 & $>19: 1$ & 97 \\
\hline 14 & cyclohexyl & $p-\mathrm{BrC}_{6} \mathrm{H}_{4}$ & $\mathrm{Me}$ & $\mathrm{Me}$ & 95 & $>19: 1$ & 97 \\
\hline 15 & $\mathrm{Ph}$ & $m-\mathrm{O}_{2} \mathrm{NC}_{6} \mathrm{H}_{4}$ & $\mathrm{H}$ & Et & 98 & $>19: 1$ & 97 \\
\hline 16 & $\mathrm{Ph}$ & $\mathrm{CO}_{2} \mathrm{Et}$ & $\mathrm{H}$ & Et & 95 & $>19: 1$ & 97 \\
\hline 17 & cyclohexyl & $p-\mathrm{BrC}_{6} \mathrm{H}_{4}$ & $\mathrm{H}$ & Et & 93 & $>19: 1$ & 97 \\
\hline 18 & ${ }^{i} \mathrm{Pr}$ & $p-\mathrm{BrC}_{6} \mathrm{H}_{4}$ & $\mathrm{H}$ & Et & 98 & $>19: 1$ & 97 \\
\hline 19 & $\mathrm{Ph}$ & $\mathrm{CO}_{2} \mathrm{Et}$ & $\mathrm{H}$ & $\mathrm{Ph}$ & 95 & $>19: 1$ & 99 \\
\hline 20 & ${ }^{i} \mathrm{Pr}$ & $p-\mathrm{BrC}_{6} \mathrm{H}_{4}$ & $\mathrm{H}$ & $\mathrm{Ph}$ & 91 & $>19: 1$ & 99 \\
\hline 21 & $\mathrm{Ph}$ & $p-\mathrm{BrC}_{6} \mathrm{H}_{4}$ & $\mathrm{H}$ & $\mathrm{Ph}$ & 87 & $>19: 1$ & 99 \\
\hline 22 & $\mathrm{Ph}$ & $p-\mathrm{O}_{2} \mathrm{NC}_{6} \mathrm{H}_{4}$ & $\mathrm{H}$ & $\mathrm{Ph}$ & 98 & $>19: 1$ & 99 \\
\hline
\end{tabular}

Very recently, Du and co-workers reported a squaramide-catalyzed highly diastereo- and enantioselective domino Michael/Mannich [3 + 2] cycloaddition reaction between 5-alkylidenerhodanine derivatives 3 and $N$ - 
(2,2,2-trifluoroethyl)isatin ketimines 33 (Scheme 14, Table 4). The reaction proceeded smoothly to yield a broad range of $\mathrm{CF}_{3}$-containing bispiro[oxindole-pyrrolidine-rhodanine]s $\mathbf{3 4}$ bearing four contiguous stereocenters, including two vicinal spiro-quaternary chiral centers, in good to excellent yields (up to 99\%) and excellent stereoselectivities (up to $>99 \%$ ee and $>99: 1 \mathrm{dr}$ ). ${ }^{55}$ However, the reaction did not occur in the case substituents $\mathrm{R}^{2}=\mathrm{Ph}$ on the rhodanine derivative 3 (entry 4, Table 4).

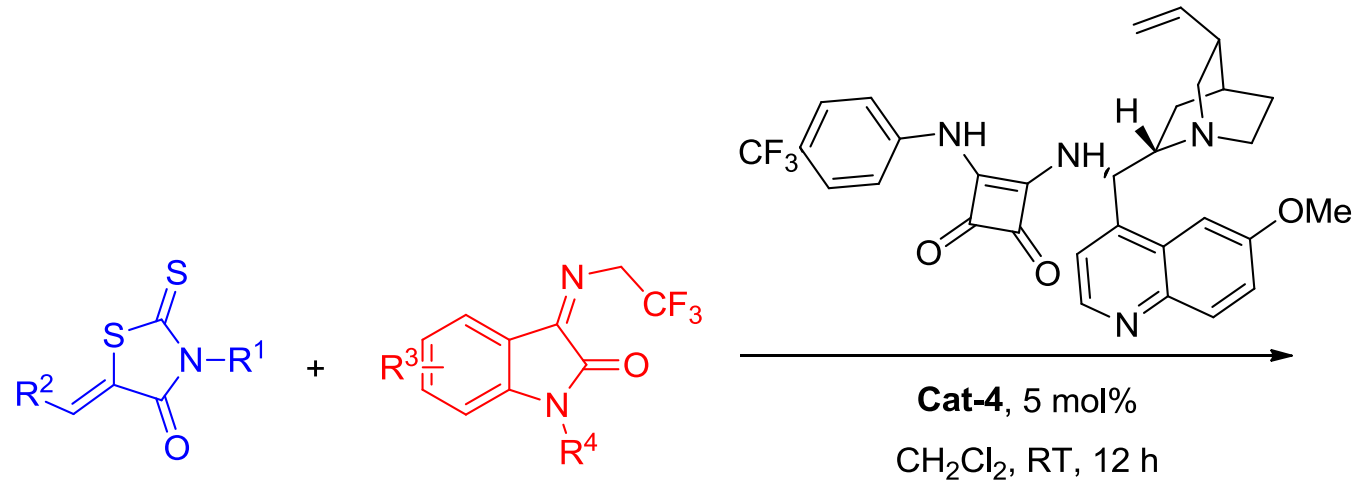

3

33

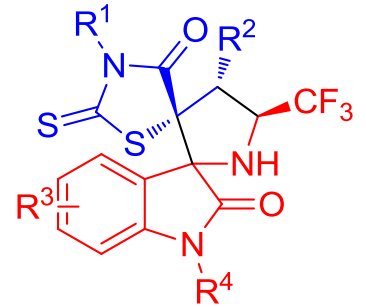

34

Scheme 14. Asymmetric Michael/Mannich [3+2] cycloaddition reaction between 5-alkylidenerhodanines 3 and $N$-(2,2,2-trifluoroethyl)isatin ketimines 33.

Table 4

\begin{tabular}{cccccccc}
\hline Entry & $\mathrm{R}^{1}$ & $\mathrm{R}^{2}$ & $\mathrm{R}^{3}$ & $\mathrm{R}^{4}$ & Yield (\%) & $\mathrm{dr}$ & ee (\%) \\
\hline 1 & $\mathrm{Ph}$ & $\mathrm{CO}_{2} \mathrm{Et}$ & $\mathrm{H}$ & $\mathrm{Bn}$ & 99 & $99: 1$ & 96 \\
2 & $\mathrm{Ph}$ & $\mathrm{Boc}$ & $\mathrm{H}$ & $\mathrm{Bn}$ & 96 & $99: 1$ & 93 \\
3 & $\mathrm{Ph}$ & $\mathrm{CN}$ & $\mathrm{H}$ & $\mathrm{Bn}$ & 95 & $92: 8$ & 83 \\
4 & $\mathrm{Ph}$ & $\mathrm{Ph}$ & $\mathrm{H}$ & $\mathrm{Bn}$ & & no reaction & \\
5 & $\mathrm{Bn}$ & $\mathrm{CO}_{2} \mathrm{Et}$ & $\mathrm{H}$ & $\mathrm{Bn}$ & 85 & $99: 1$ & 97 \\
6 & $\mathrm{cyclohexyl}$ & $\mathrm{CO}_{2} \mathrm{Et}$ & $\mathrm{H}$ & $\mathrm{Bn}$ & 94 & $99: 1$ & $>99$ \\
7 & $\mathrm{Me}$ & $\mathrm{CO}_{2} \mathrm{Et}$ & $\mathrm{H}$ & $\mathrm{Bn}$ & 93 & $98: 2$ & 95 \\
8 & $n \mathrm{Pr}$ & $\mathrm{CO}_{2} \mathrm{Et}$ & $\mathrm{H}$ & $\mathrm{Bn}$ & 97 & $99: 1$ & $>99$ \\
9 & $\mathrm{H}$ & $\mathrm{CO}_{2} \mathrm{Et}$ & $\mathrm{H}$ & $\mathrm{Bn}$ & 65 & $>99: 1$ & 57 \\
10 & $p-\mathrm{MeC}_{6} \mathrm{H}_{4}$ & $\mathrm{CO}_{2} \mathrm{Et}$ & $\mathrm{H}$ & $\mathrm{Bn}$ & 97 & $99: 1$ & 90 \\
11 & $p-\mathrm{MeOC}_{6} \mathrm{H}_{4}$ & $\mathrm{CO}_{2} \mathrm{Et}$ & $\mathrm{H}$ & $\mathrm{Bn}$ & 97 & $99: 1$ & 90 \\
12 & $p-\mathrm{FC}_{6} \mathrm{H}_{4}$ & $\mathrm{CO}_{2} \mathrm{Et}$ & $\mathrm{H}$ & $\mathrm{Bn}$ & 90 & $98: 2$ & 91 \\
13 & $p-\mathrm{ClC}_{6} \mathrm{H}_{4}$ & $\mathrm{CO}_{2} \mathrm{Et}$ & $\mathrm{H}$ & $\mathrm{Bn}$ & 98 & $99: 1$ & 90 \\
14 & $p-\mathrm{BrC}_{6} \mathrm{H}_{4}$ & $\mathrm{CO}_{2} \mathrm{Et}$ & $\mathrm{H}$ & $\mathrm{Bn}$ & 99 & $98: 2$ & 91 \\
15 & $\mathrm{Ph}_{16}$ & $\mathrm{CO}_{2} \mathrm{Et}$ & $5-\mathrm{OMe}$ & $\mathrm{Bn}$ & 99 & $99: 1$ & $>99$ \\
16 & $\mathrm{Ph}_{17}$ & $\mathrm{CO}_{2} \mathrm{Et}$ & $5-\mathrm{Me}$ & $\mathrm{Bn}$ & 99 & $99: 1$ & 93 \\
\hline
\end{tabular}


Table 4. Continued

\begin{tabular}{cccccccc}
\hline Entry & $\mathrm{R}^{1}$ & $\mathrm{R}^{2}$ & $\mathrm{R}^{3}$ & $\mathrm{R}^{4}$ & Yield (\%) & $\mathrm{dr}$ & ee (\%) \\
\hline 18 & $\mathrm{Ph}$ & $\mathrm{CO}_{2} \mathrm{Et}$ & $5-\mathrm{F}$ & $\mathrm{Bn}$ & 98 & $95: 5$ & 80 \\
19 & $\mathrm{Ph}$ & $\mathrm{CO}_{2} \mathrm{Et}$ & $5-\mathrm{Cl}$ & $\mathrm{Bn}$ & 95 & $93: 7$ & $>99$ \\
20 & $\mathrm{Ph}$ & $\mathrm{CO}_{2} \mathrm{Et}$ & $6-\mathrm{Cl}$ & $\mathrm{Bn}$ & 91 & $86: 14$ & 91 \\
21 & $\mathrm{Ph}$ & $\mathrm{CO}_{2} \mathrm{Et}$ & $5-\mathrm{Br}$ & $\mathrm{Bn}$ & 96 & $95: 5$ & 95 \\
22 & $\mathrm{Ph}$ & $\mathrm{CO}_{2} \mathrm{Et}$ & $\mathrm{H}$ & $\mathrm{H}$ & 85 & $97: 3$ & 86 \\
23 & $\mathrm{Ph}$ & $\mathrm{CO}_{2} \mathrm{Et}$ & $\mathrm{H}$ & $\mathrm{Me}$ & 92 & $99: 1$ & 89 \\
24 & $\mathrm{Ph}$ & $\mathrm{CO}_{2} \mathrm{Et}$ & $\mathrm{H}$ & allyl & 98 & $98: 2$ & 89 \\
25 & $\mathrm{Ph}$ & $\mathrm{CO}_{2} \mathrm{Et}$ & $\mathrm{H}$ & $p-\mathrm{O}_{2} \mathrm{NC}_{6} \mathrm{H}_{4}$ & 82 & $>99: 1$ & 90 \\
26 & $\mathrm{Ph}$ & $\mathrm{CO}_{2} \mathrm{Et}$ & $\mathrm{H}$ & $p-\mathrm{BrC}_{6} \mathrm{H}_{4}$ & 87 & $98: 2$ & 92 \\
\hline
\end{tabular}

\subsection{Asymmetric reactions of 5 -substituted rhodanines 4}

The first report of direct diastereo- and enantioselective Michael addition using 5-substituted rhodanines 4 with $\alpha, \beta$-unsaturated ketones 35 was reported by Ye et al. in 2012. The catalyst of choice was the chiral diamine Cat-5, which furnishes varieties of enantioenriched rhodanine derivatives $\mathbf{3 6}$ in high yields (up to 98\%) with excellent diastereoselectivities (up to 99:1 dr) and enantioselectivities (up to >99\% ee) (Scheme 15, Table 5). ${ }^{49}$<smiles>[R]C1SC(=S)N([R])C1=O</smiles>

4<smiles>[R]C=CC([R])=O</smiles>

35

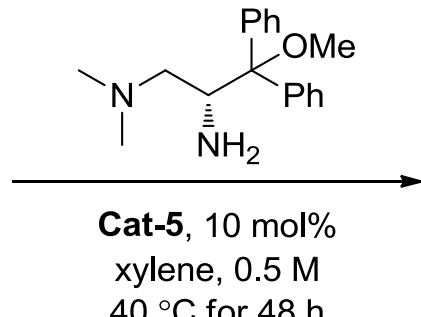

$40{ }^{\circ} \mathrm{C}$ for $48 \mathrm{~h}$<smiles>[R]C(=O)CC([R3])[C@]1([R2])SC(=S)N([R])C1=O</smiles>

36

Scheme 15. Asymmetric Michael addition of substituted rhodanines 4 with $\alpha, \beta$-unsaturated ketones 35.

Table 5

\begin{tabular}{cccccccc}
\hline Entry & $\mathrm{R}^{1}$ & $\mathrm{R}^{2}$ & $\mathrm{R}^{3}$ & $\mathrm{R}^{4}$ & Yield (\%) & $\mathrm{dr}$ & ee (\%) \\
\hline 1 & $\mathrm{Ph}$ & $\mathrm{Me}$ & $\mathrm{Ph}$ & $\mathrm{Me}$ & 95 & $99: 1$ & 96 \\
2 & $\mathrm{Ph}$ & $\mathrm{Me}$ & $p-\mathrm{MeC}_{6} \mathrm{H}_{4}$ & $\mathrm{Me}$ & 75 & $97: 3$ & 93 \\
3 & $\mathrm{Ph}$ & $\mathrm{Me}$ & $m-\mathrm{MeOC}_{6} \mathrm{H}_{4}$ & $\mathrm{Me}$ & 81 & $99: 1$ & 94 \\
4 & $\mathrm{Ph}$ & $\mathrm{Me}$ & $m-\mathrm{BrC}_{6} \mathrm{H}_{4}$ & $\mathrm{Me}$ & 92 & $99: 1$ & 93 \\
5 & $\mathrm{Ph}$ & $\mathrm{Me}$ & $p-\mathrm{ClC}_{6} \mathrm{H}_{4}$ & $\mathrm{Me}$ & 95 & $99: 1$ & 96 \\
6 & $\mathrm{Ph}$ & $\mathrm{Me}$ & $0-\mathrm{O}_{2} \mathrm{NC}_{6} \mathrm{H}_{4}$ & $\mathrm{Me}$ & 89 & $99: 1$ & 95 \\
7 & $\mathrm{Ph}$ & $\mathrm{Me}$ & $\mathrm{Naphthyl}$ & $\mathrm{Me}$ & 93 & $98: 2$ & 91 \\
8 & $\mathrm{Ph}$ & $\mathrm{Me}$ & 2-thiophenyl & $\mathrm{Me}$ & 96 & $97: 3$ & 98 \\
9 & $\mathrm{Ph}$ & $\mathrm{Me}$ & $2-\mathrm{furanyl}$ & $\mathrm{Me}$ & 84 & $99: 1$ & 95 \\
10 & $\mathrm{Ph}$ & $\mathrm{Me}$ & $\mathrm{Me}_{11}$ & $\mathrm{Me}$ & 64 & $95: 5$ & 80 \\
11 & $\mathrm{Ph}$ & $\mathrm{Me}$ & $\mathrm{PhCH}_{2} \mathrm{CH}_{2}$ & $\mathrm{Me}$ & 60 & $97: 3$ & 87 \\
\hline
\end{tabular}


Table 5. Continued

\begin{tabular}{|c|c|c|c|c|c|c|c|}
\hline Entry & $\mathrm{R}^{1}$ & $\mathrm{R}^{2}$ & $\mathrm{R}^{3}$ & $\mathrm{R}^{4}$ & Yield (\%) & $d r$ & ee $(\%)$ \\
\hline 12 & $\mathrm{Ph}$ & $\mathrm{Me}$ & $(E)-\mathrm{PhCH}=\mathrm{CH}$ & $\mathrm{Me}$ & 83 & $93: 7$ & 96 \\
\hline 13 & ${ }^{i} \mathrm{Pr}$ & $\mathrm{Me}$ & $\mathrm{Ph}$ & Me & 97 & $99: 1$ & 90 \\
\hline 14 & $\mathrm{Bn}$ & $\mathrm{Me}$ & $\mathrm{Ph}$ & $\mathrm{Me}$ & 94 & $98: 2$ & 95 \\
\hline 15 & $p-\mathrm{MeOC}_{6} \mathrm{H}_{4}$ & $\mathrm{Me}$ & $\mathrm{Ph}$ & $\mathrm{Me}$ & 98 & 99:1 & 96 \\
\hline 16 & $\mathrm{Bn}$ & $\mathrm{Me}$ & $p-\mathrm{ClC}_{6} \mathrm{H}_{4}$ & $\mathrm{Me}$ & 97 & $97: 3$ & 95 \\
\hline 17 & $p-\mathrm{MeOC}_{6} \mathrm{H}_{4}$ & $\mathrm{Me}$ & $p-\mathrm{BrC}_{6} \mathrm{H}_{4}$ & Me & 96 & $99: 1$ & 95 \\
\hline 18 & $\mathrm{Ph}$ & $\mathrm{Me}$ & $\mathrm{Ph}$ & ${ }^{n} \operatorname{Pr}$ & 82 & 99:1 & 95 \\
\hline 19 & $\mathrm{Ph}$ & Et & $\mathrm{Ph}$ & Me & 91 & $98: 2$ & 97 \\
\hline 20 & $\mathrm{Ph}$ & Et & Naphthyl & $\mathrm{Me}$ & 96 & 99:1 & 91 \\
\hline 21 & $\mathrm{Ph}$ & Et & $p-\mathrm{O}_{2} \mathrm{NC}_{6} \mathrm{H}_{4}$ & Me & 98 & $97: 3$ & 92 \\
\hline 22 & $\mathrm{Ph}$ & ${ }^{i} \mathrm{Pr}$ & $\mathrm{Ph}$ & $\mathrm{Me}$ & 68 & $93: 7$ & 71 \\
\hline 23 & $\mathrm{Ph}$ & $\mathrm{Me}$ & $\mathrm{Ph}$ & $\mathrm{Ph}$ & 82 & $>30: 1$ & 92 \\
\hline 24 & $\mathrm{Ph}$ & $\mathrm{Me}$ & $\mathrm{Ph}$ & $p-\mathrm{Br}-\mathrm{C}_{6} \mathrm{H}_{4}$ & 89 & $>30: 1$ & 93 \\
\hline 25 & $\mathrm{Ph}$ & $\mathrm{Me}$ & $p-\mathrm{MeC}_{6} \mathrm{H}_{4}$ & $\mathrm{Ph}$ & 93 & $>30: 1$ & 93 \\
\hline 26 & $\mathrm{Ph}$ & $\mathrm{Me}$ & cyclopenten & ne (35) & 82 & $5: 1$ & 94 \\
\hline 27 & $\mathrm{Ph}$ & $\mathrm{Me}$ & cyclohexen & ne (35) & 89 & $6: 1$ & $>99$ \\
\hline 28 & $\mathrm{Ph}$ & $\mathrm{Me}$ & cyclohepten & one (35) & 93 & $1: 1$ & $>99$ \\
\hline
\end{tabular}

In 2014, Wang and co-workers reported a highly efficient quinine-catalyzed $\alpha$-amination reaction between 5-substituted rhodanines 4 as nucleophiles with diethyl azodicarboxylate 37 (Scheme 16, Table 6). The method was applied to access a broad range of chiral N,S-acetals 38 in high yields (up to 99\%) and excellent enantioselectivities (ee up to $96 \%$ ). In addition, yield and selectivity for synthesis on gram scale were perfectly maintained for this method. ${ }^{56}$<smiles>[R]C1SC(=S)N([R])C1=O</smiles>

4<smiles>CCOCN=NC(=O)OCC</smiles>

37

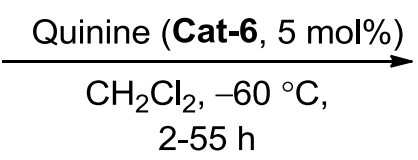

2-55 h<smiles>[R]N1C(=O)[C@]([R2])(N(C(=O)OCC)C(=O)OCC)SC1=S</smiles>

38

Scheme 16. Asymmetric $\alpha$-amination of 5-substituted rhodanines 4 with diethyl azodicarboxylate 37.

Quite recently, Wu and co-workers have developed a chiral cyclohexane-based phosphine type catalyst Cat-7 for enantioselective $\gamma$-additions of rhodanines 4 to allenoates 39 (Scheme 17, Table 7). ${ }^{57}$ The method was very useful for the construction of chiral rhodanine derivatives $\mathbf{4 0}$ containing tertiary chiral carbon centers in good yields (up to 83\%) and high enantioselectivities (up to 96\%). However the method was unsuccessful for the $\gamma$-additions of benzyl penta-2,3-dienoate and benzyl but-2-ynoate electrophiles. 
Table 6

\begin{tabular}{|c|c|c|c|c|c|c|c|c|c|}
\hline Entry & $\mathrm{R}^{1}$ & $\mathrm{R}^{2}$ & Yield (\%) & ee (\%) & Entry & $\mathrm{R}^{1}$ & $\mathrm{R}^{2}$ & Yield (\%) & ee (\%) \\
\hline 1 & $\mathrm{Ph}$ & $\mathrm{Me}$ & 99 & 95 & 12 & $\mathrm{Ph}$ & $o-\mathrm{Cl}-\mathrm{Bn}$ & 93 & 94 \\
\hline 2 & $\mathrm{Ph}$ & Et & 95 & 96 & 13 & $\mathrm{Ph}$ & $3,4-\mathrm{Cl}_{2}-\mathrm{Bn}$ & 90 & 95 \\
\hline 3 & $\mathrm{Ph}$ & $i-\operatorname{Pr}$ & 99 & 91 & 14 & $\mathrm{Me}$ & $p-\mathrm{MeOC}_{6} \mathrm{H}_{4}$ & 98 & 95 \\
\hline 4 & $\mathrm{Ph}$ & $n-\mathrm{Bu}$ & 98 & 94 & 15 & $\mathrm{Me}$ & $p-\mathrm{MeC}_{6} \mathrm{H}_{4}$ & 95 & 95 \\
\hline 5 & $\mathrm{Ph}$ & allyl & 90 & 94 & 16 & $\mathrm{Me}$ & $p-\mathrm{FC}_{6} \mathrm{H}_{4}$ & 99 & 94 \\
\hline 6 & $\mathrm{Ph}$ & $\mathrm{MeSCH}_{2} \mathrm{CH}_{2}$ & 92 & 94 & 17 & $\mathrm{Me}$ & $p-\mathrm{BrC}_{6} \mathrm{H}_{4}$ & 96 & 94 \\
\hline 7 & $\mathrm{Ph}$ & $\mathrm{Ph}$ & 91 & 81 & 18 & $\mathrm{Me}$ & $p-\mathrm{IC}_{6} \mathrm{H}_{4}$ & 98 & 94 \\
\hline 8 & $\mathrm{Ph}$ & $\mathrm{Bn}$ & 98 & 94 & 19 & $\mathrm{Me}$ & $m-\mathrm{MeC}_{6} \mathrm{H}_{4}$ & 95 & 95 \\
\hline 9 & $\mathrm{Ph}$ & o-Me-Bn & 96 & 89 & 20 & $\mathrm{Me}$ & $m-\mathrm{BrC}_{6} \mathrm{H}_{4}$ & 96 & 93 \\
\hline 10 & $\mathrm{Ph}$ & $m-\mathrm{Br}-\mathrm{Bn}$ & 91 & 92 & 21 & $\mathrm{Me}$ & $3,5-\left(\mathrm{CF}_{3}\right)_{2} \mathrm{C}_{6} \mathrm{H}_{3}$ & 99 & 88 \\
\hline 11 & $\mathrm{Ph}$ & $p$-Me-Bn & 97 & 95 & & & & & \\
\hline
\end{tabular}<smiles>[R]C1SC(=S)N([R])C1=O</smiles>

4<smiles>C=CC(=O)OCC</smiles>

39

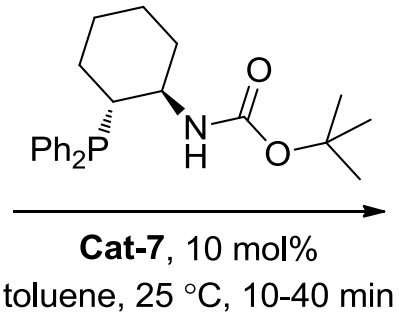

toluene, $25^{\circ} \mathrm{C}, 10-40 \mathrm{~min}$<smiles>[R9]OC(=O)/C=C/C[C@]1([R2])SC(=S)N([R7])C1=O</smiles>

40

Scheme 17. Enantioselective $\gamma$-additions of rhodanines 4 to allenoates 39.

The same group further developed quinine-based tertiary amine-thiourea catalyst Cat-8 for the enantioselective Mannich reaction of rhodanines 4 with isatin derived ketimines 41 (Scheme 18, Table 8). ${ }^{58}$ The reported reaction works well with 2 mol\% catalyst loading to obtain 3,3-disubstituted oxindoles 42 with vicinal tetrasubstituted stereocenters with excellent yields (up to 99\%), diastereoselectivities (up to 99:1) and high enantioselectivities (up to $97 \%$ ee). However, isopropyl substitution at 5-position of rhodanines 4 was found to be inactive for the enantioselective Mannich reaction under similar reaction conditions (Entry 9 , Table 8). The result was explained with steric hindrance. 
Table 7

\begin{tabular}{|c|c|c|c|c|c|c|c|c|c|c|c|}
\hline Entry & $\mathrm{R}^{1}$ & $\mathrm{R}^{2}$ & $\mathrm{R}^{3}$ & $\begin{array}{c}\text { Yield } \\
(\%)\end{array}$ & $\begin{array}{l}\text { ee } \\
(\%)\end{array}$ & Entry & $\mathrm{R}^{1}$ & $\mathrm{R}^{2}$ & $\mathrm{R}^{3}$ & $\begin{array}{c}\text { Yield } \\
(\%)\end{array}$ & $\begin{array}{l}\text { ee } \\
(\%)\end{array}$ \\
\hline 1 & $\mathrm{Ph}$ & $\mathrm{Me}$ & $\mathrm{Bn}$ & 78 & 94 & 11 & $\mathrm{Ph}$ & ${ }^{i} \operatorname{Pr}$ & $\mathrm{Bn}$ & 62 & 80 \\
\hline 2 & $\mathrm{Bn}$ & $\mathrm{Me}$ & $\mathrm{Bn}$ & 79 & 92 & 12 & $\mathrm{Ph}$ & ${ }^{n} \mathrm{Pr}$ & $\mathrm{Bn}$ & 72 & 86 \\
\hline 3 & $p-\mathrm{MeOC}_{6} \mathrm{H}_{4}$ & Me & $\mathrm{Bn}$ & 74 & 95 & 13 & $\mathrm{Ph}$ & ${ }^{n} \mathrm{Bu}$ & $\mathrm{Bn}$ & 71 & 87 \\
\hline 4 & $p-\mathrm{FC}_{6} \mathrm{H}_{4}$ & Me & $\mathrm{Bn}$ & 83 & 89 & 14 & $\mathrm{Ph}$ & ${ }^{t} \mathrm{Bu}$ & $\mathrm{Bn}$ & 61 & 79 \\
\hline 5 & $p-\mathrm{ClC}_{6} \mathrm{H}_{4}$ & $\mathrm{Me}$ & $\mathrm{Bn}$ & 82 & 90 & 15 & $\mathrm{Ph}$ & $\mathrm{Me}$ & $\mathrm{Ph}$ & 68 & 82 \\
\hline 6 & $p-\mathrm{BrC}_{6} \mathrm{H}_{4}$ & $\mathrm{Me}$ & $\mathrm{Bn}$ & 81 & 90 & 16 & $\mathrm{Ph}$ & $\mathrm{Me}$ & $\mathrm{CHPh}_{2}$ & 76 & 94 \\
\hline 7 & $p-\mathrm{MeC}_{6} \mathrm{H}_{4}$ & Me & $\mathrm{Bn}$ & 77 & 93 & 17 & $\mathrm{Ph}$ & $\mathrm{Me}$ & 9-fluorenyl & 74 & 86 \\
\hline 8 & $3,5-\left(\mathrm{CF}_{3}\right)_{2} \mathrm{C}_{6} \mathrm{H}_{3}$ & Me & $\mathrm{Bn}$ & 81 & 96 & \multirow{3}{*}{18} & \multirow{3}{*}{$\mathrm{Ph}$} & \multirow{3}{*}{$\mathrm{Me}$} & \multirow{3}{*}{$\begin{array}{l}\text { 5-dibenzo- } \\
\text { cycloheptyl }\end{array}$} & \multirow{3}{*}{77} & \multirow{3}{*}{93} \\
\hline 9 & $\mathrm{Ph}$ & $\mathrm{Bn}$ & $\mathrm{Bn}$ & 76 & 91 & & & & & & \\
\hline 10 & $\mathrm{Ph}$ & Et & $\mathrm{Bn}$ & 75 & 93 & & & & & & \\
\hline
\end{tabular}<smiles>[R]C1SC(=S)N([R])C1=O</smiles>

4

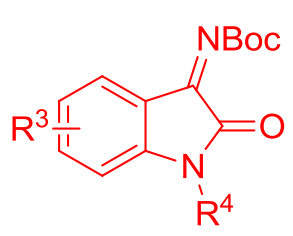

41

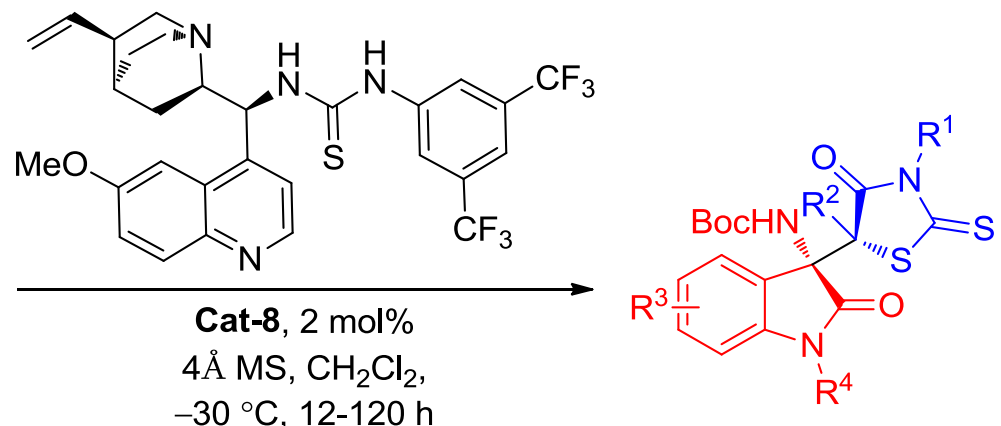

42

Scheme 18. Asymmetric Mannich reaction of rhodanines 4 with isatin-derived ketimines 41 .

Table 8

\begin{tabular}{|c|c|c|c|c|c|c|}
\hline Entry & $\mathrm{R}^{1}$ & $\mathrm{R}^{2}$ & $\mathrm{R}^{3}$ & Yield (\%) & $d r$ & ee (\%) \\
\hline 1 & $\mathrm{Ph}$ & $\mathrm{Me}$ & $\mathrm{H}$ & 99 & $99: 1$ & 94 \\
\hline 2 & $p-\mathrm{Br}-\mathrm{C}_{6} \mathrm{H}_{4}$ & $\mathrm{Me}$ & $\mathrm{H}$ & 99 & 99:1 & 94 \\
\hline 3 & $p-\mathrm{MeO}-\mathrm{C}_{6} \mathrm{H}_{4}$ & $\mathrm{Me}$ & $\mathrm{H}$ & 91 & 99:1 & 93 \\
\hline 4 & $\mathrm{Bn}$ & $\mathrm{Me}$ & $\mathrm{H}$ & 43 & $99: 1$ & 97 \\
\hline 5 & cyclohexyl & $\mathrm{Me}$ & $\mathrm{H}$ & 53 & $98: 2$ & 90 \\
\hline 6 & $\mathrm{Ph}$ & Et & $\mathrm{H}$ & 95 & 99:1 & 93 \\
\hline 7 & $\mathrm{Ph}$ & ${ }^{n} \operatorname{Pr}$ & $\mathrm{H}$ & 90 & $99: 1$ & 96 \\
\hline 8 & $\mathrm{Ph}$ & ${ }^{n} \mathrm{Bu}$ & $\mathrm{H}$ & 97 & 99:1 & 93 \\
\hline 9 & $\mathrm{Ph}$ & ${ }^{i} \operatorname{Pr}$ & $\mathrm{H}$ & \multicolumn{3}{|c|}{ no reaction } \\
\hline 10 & $\mathrm{Ph}$ & $\mathrm{Bn}$ & $\mathrm{H}$ & 99 & 99:1 & 89 \\
\hline
\end{tabular}


Table 8. Continued

\begin{tabular}{ccccccc}
\hline Entry & $\mathrm{R}^{1}$ & $\mathrm{R}^{2}$ & $\mathrm{R}^{3}$ & Yield (\%) & $\mathrm{dr}$ & ee (\%) \\
\hline 11 & $\mathrm{Ph}$ & $\mathrm{Me}$ & $5-\mathrm{NO}_{2}$ & 99 & $99: 1$ & 36 \\
12 & $\mathrm{Ph}$ & $\mathrm{Me}$ & $5-\mathrm{Br}$ & 99 & $98: 2$ & 74 \\
13 & $\mathrm{Ph}$ & $\mathrm{Me}$ & $5-\mathrm{Me}$ & 98 & $99: 1$ & 93 \\
14 & $\mathrm{Ph}$ & $\mathrm{Me}$ & $5-\mathrm{MeO}$ & 99 & $99: 1$ & 93 \\
15 & $\mathrm{Ph}$ & $\mathrm{Me}$ & $6-\mathrm{F}$ & 99 & $99: 1$ & 94 \\
16 & $\mathrm{Ph}$ & $\mathrm{Me}$ & $6-\mathrm{Cl}$ & 96 & $99: 1$ & 89 \\
17 & $\mathrm{Ph}$ & $\mathrm{Me}$ & $6-\mathrm{Br}$ & 98 & $99: 1$ & 88 \\
18 & $\mathrm{Ph}$ & $\mathrm{Me}$ & $6-\mathrm{MeO}$ & 95 & $99: 1$ & 96 \\
19 & $\mathrm{Ph}$ & $\mathrm{Me}$ & $7-\mathrm{F}$ & 97 & $99: 1$ & 87 \\
20 & $\mathrm{Ph}$ & $\mathrm{Me}$ & $7-\mathrm{Cl}$ & 99 & $99: 1$ & 84 \\
21 & $\mathrm{Ph}$ & $\mathrm{Me}$ & $7-\mathrm{Br}$ & 99 & $99: 1$ & 81 \\
22 & $\mathrm{Ph}$ & $\mathrm{Me}$ & $7-\mathrm{Me}$ & 98 & $99: 1$ & 90 \\
\hline
\end{tabular}

$\mathrm{Li}$ and co-workers reported the same reactions of rhodanines 4 with isatin derived ketimines 41 . Interestingly, they observed an excellent reaction rate and stereoselectivity when the reaction was performed in presence of 1 mol\% chiral squaramide catalyst Cat-9 at room temperature (Scheme 19, Table 9). ${ }^{59}$

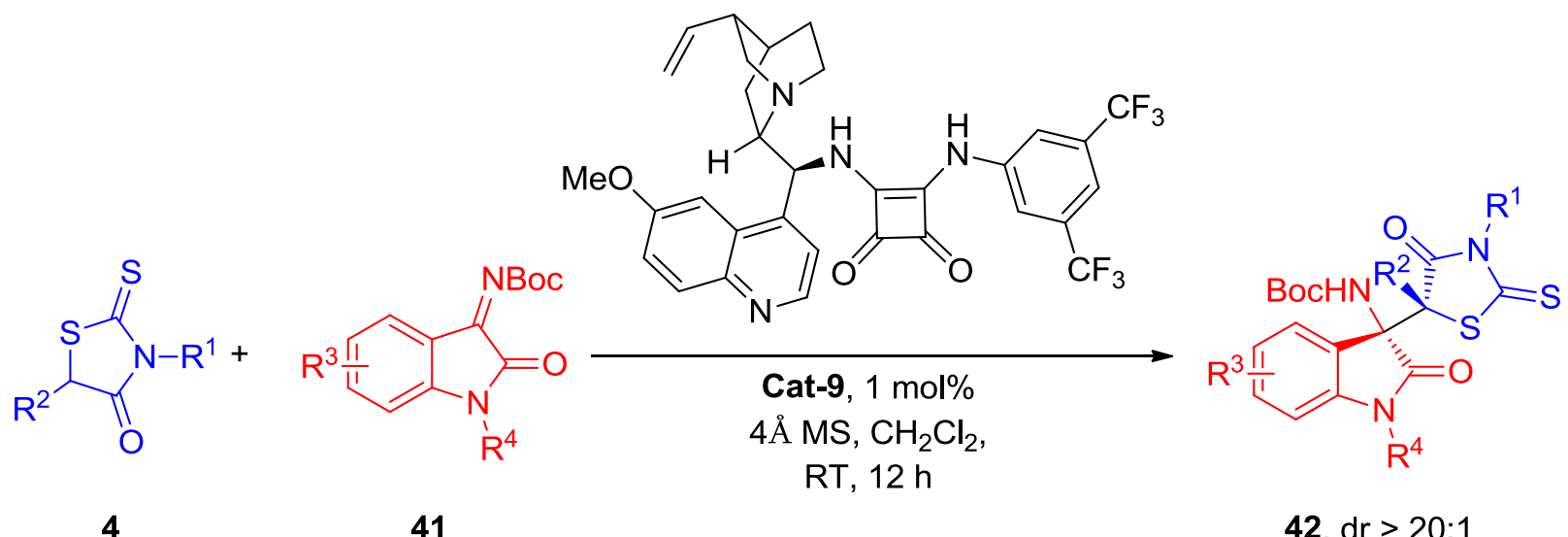

Scheme 19. Asymmetric Mannich reaction of rhodanines 4 with isatin-derived ketimines 41.

A variety of chiral 3-substituted-3-amino-2-oxindoles $\mathbf{4 2}$ containing two contiguous quaternary stereocenters were obtained in high yields (yield up to $96 \%$ ) with excellent stereoselectivities ( $d r>20: 1$ and ee up to $98 \%$ ). 
Table 9

\begin{tabular}{|c|c|c|c|c|c|c|c|}
\hline Entry & $\mathrm{R}^{1}$ & $\mathrm{R}^{2}$ & $\mathrm{R}^{3}$ & $\mathrm{R}^{4}$ & Yield (\%) & $\mathrm{dr}$ & ee $(\%)$ \\
\hline 1 & $\mathrm{Ph}$ & $\mathrm{Me}$ & $\mathrm{H}$ & $\mathrm{Bn}$ & 95 & $>20: 1$ & 96 \\
\hline 2 & $\mathrm{Ph}$ & $\mathrm{Me}$ & $5-F$ & $\mathrm{Bn}$ & 92 & $>20: 1$ & 96 \\
\hline 3 & $\mathrm{Ph}$ & $\mathrm{Me}$ & $5-\mathrm{Cl}$ & $\mathrm{Bn}$ & 90 & $>20: 1$ & 94 \\
\hline 4 & $\mathrm{Ph}$ & $\mathrm{Me}$ & $5-\mathrm{Br}$ & $\mathrm{Bn}$ & 93 & $>20: 1$ & 94 \\
\hline 5 & $\mathrm{Ph}$ & $\mathrm{Me}$ & $5-\mathrm{Me}$ & $\mathrm{Bn}$ & 91 & $>20: 1$ & 96 \\
\hline 6 & $\mathrm{Ph}$ & $\mathrm{Me}$ & $6-\mathrm{Cl}$ & $\mathrm{Bn}$ & 87 & $>20: 1$ & 90 \\
\hline 7 & $\mathrm{Ph}$ & $\mathrm{Me}$ & $6-\mathrm{Br}$ & $\mathrm{Bn}$ & 92 & $>20: 1$ & 94 \\
\hline 8 & $\mathrm{Ph}$ & $\mathrm{Me}$ & $7-F$ & $\mathrm{Bn}$ & 94 & $>20: 1$ & 92 \\
\hline 9 & $\mathrm{Ph}$ & $\mathrm{Me}$ & $7-\mathrm{Cl}$ & $\mathrm{Bn}$ & 83 & $>20: 1$ & 90 \\
\hline 10 & $\mathrm{Ph}$ & $\mathrm{Me}$ & $\mathrm{H}$ & allyl & 93 & $>20: 1$ & 90 \\
\hline 11 & $\mathrm{Ph}$ & $\mathrm{Me}$ & $\mathrm{H}$ & $\mathrm{Me}$ & 96 & $>20: 1$ & 93 \\
\hline 12 & $\mathrm{Ph}$ & $\mathrm{Me}$ & $\mathrm{H}$ & $\mathrm{Ph}$ & 95 & $>20: 1$ & 82 \\
\hline 13 & $p-\mathrm{ClC}_{6} \mathrm{H}_{4}$ & $\mathrm{Me}$ & $\mathrm{H}$ & $\mathrm{Bn}$ & 92 & $>20: 1$ & 94 \\
\hline 14 & $p-\mathrm{BrC}_{6} \mathrm{H}_{4}$ & Me & $\mathrm{H}$ & $\mathrm{Bn}$ & 94 & $>20: 1$ & 94 \\
\hline 15 & $p-\mathrm{MeC}_{6} \mathrm{H}_{4}$ & $\mathrm{Me}$ & $\mathrm{H}$ & $\mathrm{Bn}$ & 90 & $>20: 1$ & 98 \\
\hline 16 & $m-\mathrm{FC}_{6} \mathrm{H}_{4}$ & $\mathrm{Me}$ & $\mathrm{H}$ & $\mathrm{Bn}$ & 95 & $>20: 1$ & 93 \\
\hline 17 & $o-\mathrm{ClC}_{6} \mathrm{H}_{4}$ & Me & $\mathrm{H}$ & $\mathrm{Bn}$ & 94 & $>20: 1$ & 94 \\
\hline 18 & $m-\mathrm{MeC}_{6} \mathrm{H}_{4}$ & $\mathrm{Me}$ & $\mathrm{H}$ & $\mathrm{Bn}$ & 95 & $>20: 1$ & 96 \\
\hline 19 & $o-\mathrm{MeC}_{6} \mathrm{H}_{4}$ & $\mathrm{Me}$ & $\mathrm{H}$ & $\mathrm{Bn}$ & 91 & $>20: 1$ & 96 \\
\hline 20 & $\mathrm{Bn}$ & $\mathrm{Me}$ & $\mathrm{H}$ & $\mathrm{Bn}$ & 92 & $>20: 1$ & 98 \\
\hline 21 & Et & $\mathrm{Me}$ & $\mathrm{H}$ & $\mathrm{Bn}$ & 81 & $>20: 1$ & 96 \\
\hline 22 & ${ }^{i} \mathrm{Pr}$ & $\mathrm{Me}$ & $\mathrm{H}$ & $\mathrm{Bn}$ & 86 & $>20: 1$ & 98 \\
\hline 23 & $\mathrm{Ph}$ & Et & $\mathrm{H}$ & $\mathrm{Bn}$ & 92 & $>20: 1$ & 90 \\
\hline 24 & $\mathrm{Ph}$ & $\mathrm{Bn}$ & $\mathrm{H}$ & $\mathrm{Bn}$ & 90 & $>20: 1$ & 96 \\
\hline
\end{tabular}

\subsection{Asymmetric reactions of thiazol-4(5H)-ones (5)}

In 2013, Palomo's group developed the first catalytic Michael reaction of thiazol-4(5H)-ones 5 with aromatic and aliphatic nitroolefins 43 using a bifunctional Brønsted base catalyst Cat-10 (Scheme 20, Table 10). ${ }^{60}$ The method reflects the direct formation of a quaternary chiral carbon centre with high stereoselectivities (dr up to $>95: 5$ and ee up to $98 \%$ ) and yields (up to 96\%). However, comparitively low yields were observed in the reaction with aliphatic nitroolefins without affecting the stereoselectivities (entries 14-16). 


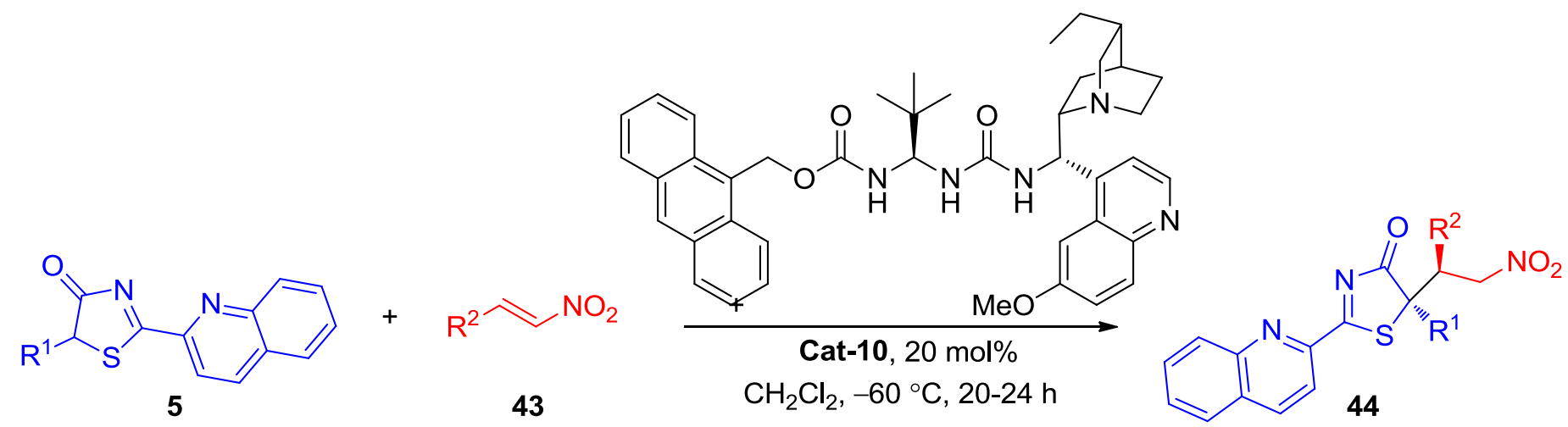

Scheme 20. Asymmetric Michael reaction of thiazol-4(5H)-ones 5 with nitroolefins 43.

Table 10

\begin{tabular}{|c|c|c|c|c|c|}
\hline Entry & $\mathrm{R}^{1}$ & $\mathrm{R}^{2}$ & Yield (\%) & $\mathrm{dr}$ & ee $(\%)$ \\
\hline 1 & $\mathrm{Me}$ & $\mathrm{Ph}$ & 93 & $95: 5$ & 96 \\
\hline 2 & Me & $m-\mathrm{MeC}_{6} \mathrm{H}_{4}$ & 90 & $>95: 5$ & 91 \\
\hline 3 & Me & $p-\mathrm{MeOC}_{6} \mathrm{H}_{4}$ & 77 & $>95: 5$ & 92 \\
\hline 4 & Me & $m-\mathrm{MeOC}_{6} \mathrm{H}_{4}$ & 77 & $>95: 5$ & 94 \\
\hline 5 & Me & $p-\mathrm{FC}_{6} \mathrm{H}_{4}$ & 74 & $>95: 5$ & 90 \\
\hline 6 & $\mathrm{Me}$ & $p-\mathrm{BrC}_{6} \mathrm{H}_{4}$ & 79 & $>95: 5$ & 92 \\
\hline 7 & Me & $p-\mathrm{ClC}_{6} \mathrm{H}_{4}$ & 75 & $>95: 5$ & 92 \\
\hline 8 & $\mathrm{Me}$ & $p-\mathrm{O}_{2} \mathrm{NC}_{6} \mathrm{H}_{4}$ & 68 & $>95: 5$ & 80 \\
\hline 9 & Me & $p-\mathrm{NCC}_{6} \mathrm{H}_{4}$ & 72 & $>95: 5$ & 86 \\
\hline 10 & Me & 2-naphthyl & 82 & $>92: 8$ & 94 \\
\hline 11 & $\mathrm{Me}$ & 2-furyl & 96 & $>92: 8$ & 91 \\
\hline 12 & Me & 3-furyl & 95 & $>92: 8$ & 89 \\
\hline 13 & Me & 2-thienyl & 93 & $>92: 8$ & 92 \\
\hline 14 & Me & $i-\mathrm{Pr}$ & 47 & $>95: 5$ & 91 \\
\hline 15 & $\mathrm{Me}$ & $n-\operatorname{Pr}$ & 42 & $>95: 5$ & 76 \\
\hline 16 & Me & cyclohexyl & 40 & $>95: 5$ & 91 \\
\hline 17 & Et & $\mathrm{Ph}$ & 95 & $>95: 5$ & 97 \\
\hline 18 & Et & $p-\mathrm{MeC}_{6} \mathrm{H}_{4}$ & 87 & $>95: 5$ & 91 \\
\hline 19 & Et & $p-\mathrm{MeOC}_{6} \mathrm{H}_{4}$ & 95 & $>95: 5$ & 97 \\
\hline 20 & Et & $p-\mathrm{BrC}_{6} \mathrm{H}_{4}$ & 81 & $>95: 5$ & 97 \\
\hline 21 & $n$-hexyl & $\mathrm{Ph}$ & 96 & $>95: 5$ & 92 \\
\hline 22 & $n$-hexyl & $p-\mathrm{MeC}_{6} \mathrm{H}_{4}$ & 96 & $>95: 5$ & 98 \\
\hline 23 & $\mathrm{Bn}$ & $\mathrm{Ph}$ & 90 & $>95: 5$ & 92 \\
\hline
\end{tabular}

In 2016, Jiang and co-workers performed similar reactions of thiazol-4(5H)-ones 5 with sevaral electrondeficient alkenes 43, 46, 48 and 50 using a dipeptide-based multifunctional Brønsted base organocatalyst Cat11. ${ }^{61}$ They found the formation of several 1,4-sulfur-bridged piperidinone structural motifs 45, 47, 49 and 51 with various hetero-quaternary stereogenic centers through the asymmetric [4+2] annulation reaction. The 
origin of enantio- and chemoselectivity were supported by density functional theory studies. Initially the substrates scope for the asymmetric [4+2] annulation reaction of thiazol-4(5H)-ones 5 were checked with nitroolefins 43 (Scheme 21, Table 11). The reactions proceeded well under the optimized reaction conditions with excellent diastereoselectivities ( $d r>20: 1$ ), enantioselectivities (ee up to 98\%) with high yields (up to $89 \%$ ).

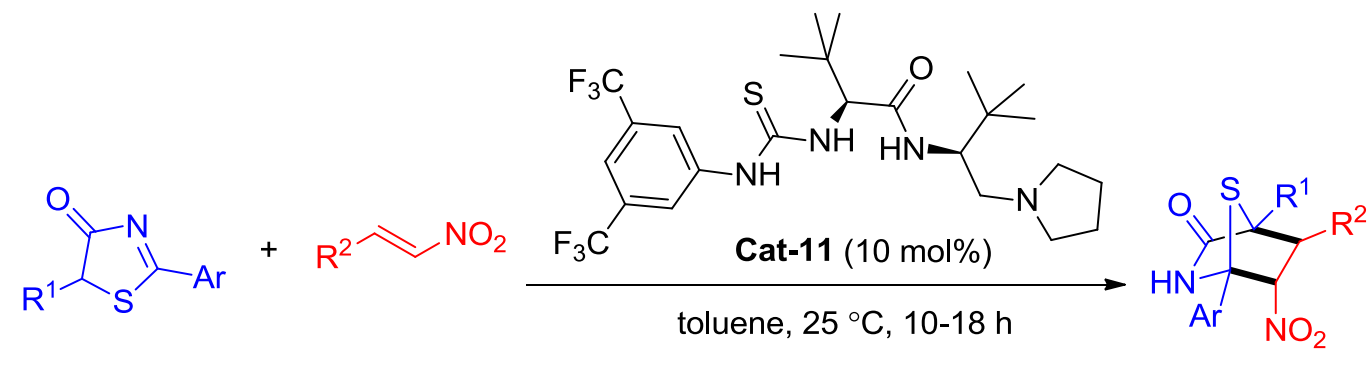

5

43

45

Scheme 21. Asymmetric [4+2] annulation reaction of thiazol-4(5H)-ones 5 with nitroolefins 43.

\section{Table 11}

\begin{tabular}{ccccccc}
\hline Entry & $\mathrm{R}^{1}$ & $\mathrm{R}^{2}$ & Ar & Yield (\%) & $\mathrm{dr}$ & ee (\%) \\
\hline 1 & $\mathrm{Me}$ & $\mathrm{Ph}$ & 2-quinolyl & 83 & $>20: 1$ & 95 \\
2 & $\mathrm{Me}$ & $p-\mathrm{FC}_{6} \mathrm{H}_{4}$ & 2-quinolyl & 78 & $>20: 1$ & 97 \\
3 & $\mathrm{Me}$ & $p-\mathrm{BrC}_{6} \mathrm{H}_{4}$ & 2-quinolyl & 84 & $>20: 1$ & 96 \\
4 & $\mathrm{Me}$ & $p-\mathrm{ClC}_{6} \mathrm{H}_{4}$ & 2-quinolyl & 89 & $>20: 1$ & 94 \\
5 & $\mathrm{Me}$ & $m-\mathrm{ClC}_{6} \mathrm{H}_{4}$ & 2-quinolyl & 85 & $>20: 1$ & 92 \\
6 & $\mathrm{Me}$ & ${ }^{-}-\mathrm{ClC}_{6} \mathrm{H}_{4}$ & 2-quinolyl & 82 & $>20: 1$ & 95 \\
7 & $\mathrm{Me}$ & $p-\mathrm{MeC}_{6} \mathrm{H}_{4}$ & 2-quinolyl & 72 & $>20: 1$ & 95 \\
8 & $\mathrm{Me}$ & $m-\mathrm{MeC}_{6} \mathrm{H}_{4}$ & 2-quinolyl & 76 & $>20: 1$ & 96 \\
9 & $\mathrm{Me}$ & $o-\mathrm{MeC}_{6} \mathrm{H}_{4}$ & 2-quinolyl & 82 & $>20: 1$ & 93 \\
10 & $\mathrm{Me}$ & 2-naphthyl & 2-quinolyl & 88 & $>20: 1$ & 95 \\
11 & $\mathrm{Me}$ & 2-furanyl & 2-quinolyl & 76 & $>20: 1$ & 94 \\
12 & $\mathrm{Me}$ & cyclohexyl & 2-quinolyl & 41 & $>20: 1$ & 82 \\
13 & $\mathrm{Et}$ & $\mathrm{Ph}$ & 2-quinolyl & 55 & $>20: 1$ & 98 \\
14 & $\mathrm{Me}$ & $\mathrm{Ph}$ & $p$-BrC $\mathrm{H}_{4}$ & 45 & $>20: 1$ & 90 \\
15 & $\mathrm{Me}$ & $\mathrm{Ph}$ & 3-pyridinyl & 76 & $>20: 1$ & 95 \\
\hline
\end{tabular}

Next the substrate scope was extended by the coupling of thiazol-4(5H)-ones 5 with trans-4-oxo-4arylbutenones 46 and trans-4-oxo-4-arylbutenoates 48 (Scheme 22 and 23, Table 12 and 13). 


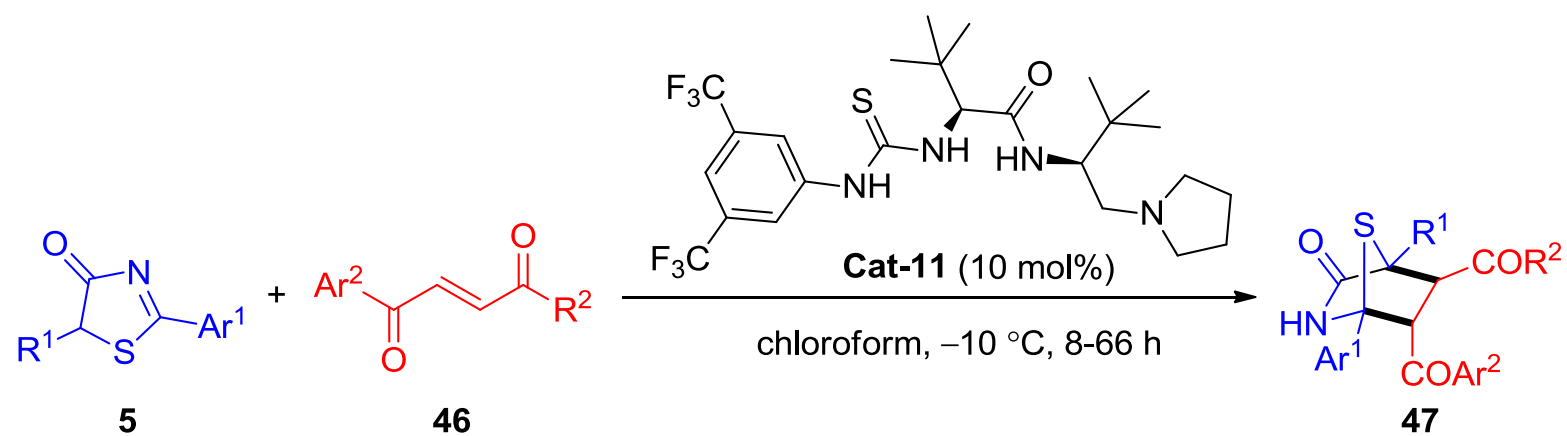

Scheme 22. Asymmetric [4+2] annulation of thiazol-4(5H)-ones 5 with trans-4-oxo-4-arylbutenones 46.

Table 12

\begin{tabular}{cccccccc}
\hline Entry & $\mathrm{R}^{1}$ & $\mathrm{R}^{2}$ & $\mathrm{Ar}^{1}$ & $\mathrm{Ar}^{2}$ & Yield (\%) & $\mathrm{dr}$ & ee (\%) \\
\hline 1 & $\mathrm{Me}$ & $\mathrm{Me}$ & 2-quinolyl & $\mathrm{Ph}$ & 88 & $>20: 1$ & 98 \\
2 & $\mathrm{Me}$ & $\mathrm{Me}$ & 2-quinolyl & $p-\mathrm{CF}_{3}-\mathrm{C}_{6} \mathrm{H}_{4}$ & 90 & $>20: 1$ & 98 \\
3 & $\mathrm{Me}$ & $\mathrm{Me}$ & 2-quinolyl & $p$ - $\mathrm{Br}_{6} \mathrm{C}_{6} \mathrm{H}_{4}$ & 91 & $>20: 1$ & 99 \\
4 & $\mathrm{Me}$ & $\mathrm{Me}$ & 2-quinolyl & $p-\mathrm{Cl}_{6} \mathrm{C}_{6} \mathrm{H}_{4}$ & 80 & $>20: 1$ & 97 \\
5 & $\mathrm{Me}$ & $\mathrm{Me}$ & 2-quinolyl & 3,4-Cl${ }_{2} \mathrm{C}_{6} \mathrm{H}_{4}$ & 95 & $>20: 1$ & 94 \\
6 & $\mathrm{Me}$ & $\mathrm{Me}$ & 2-quinolyl & $p-\mathrm{MeOC}_{6} \mathrm{H}_{4}$ & 87 & $>20: 1$ & 97 \\
7 & $\mathrm{Me}$ & $\mathrm{Me}$ & 2-quinolyl & $m-\mathrm{MeOC}_{6} \mathrm{H}_{4}$ & 82 & $>20: 1$ & 96 \\
8 & $\mathrm{Me}$ & $\mathrm{Me}$ & 2-quinolyl & o-MeOC $\mathrm{H}_{4}$ & 64 & $>20: 1$ & 94 \\
9 & $\mathrm{Me}$ & $\mathrm{Me}$ & 2-quinolyl & 2-thienyl & 98 & $>20: 1$ & 99 \\
10 & $\mathrm{Me}$ & $\mathrm{Me}$ & 2-quinolyl & 2-furyl & 92 & $>20: 1$ & 99 \\
11 & $\mathrm{Me}$ & $\mathrm{Et}$ & 2-quinolyl & $\mathrm{Ph}$ & 90 & $>20: 1$ & 97 \\
12 & $\mathrm{Me}$ & $\mathrm{Me}$ & 2-pyridinyl & $\mathrm{Ph}$ & 87 & $>20: 1$ & 98 \\
13 & $\mathrm{Me}$ & $\mathrm{Me}$ & 3-pyridinyl & $\mathrm{Ph}$ & 86 & $>20: 1$ & 98 \\
14 & $\mathrm{Me}$ & $\mathrm{Me}$ & 2-thienyl & $\mathrm{Ph}$ & 70 & $>20: 1$ & 99 \\
15 & $\mathrm{Me}$ & $\mathrm{Me}$ & 2-furyl & $\mathrm{Ph}$ & 75 & $>20: 1$ & 99 \\
16 & $\mathrm{Me}$ & $\mathrm{Me}$ & $p$-BrC $\mathrm{H}_{4}$ & $\mathrm{Ph}$ & 54 & $>20: 1$ & 99 \\
17 & $\mathrm{Et}$ & $\mathrm{Me}$ & 2-quinolyl & $\mathrm{Ph}$ & 72 & $>20: 1$ & 97 \\
18 & $\mathrm{Bn}$ & $\mathrm{Me}$ & 2-quinolyl & $\mathrm{Ph}$ & 90 & $>20: 1$ & 98 \\
\hline
\end{tabular}

The coupling reaction with trans-4-oxo-4-arylbutenones occurs in general at $-10{ }^{\circ} \mathrm{C}$ whereas the reaction with trans-4-oxo-4-arylbutenoates proceeded preferably at $-30^{\circ} \mathrm{C}$ for the best results. 


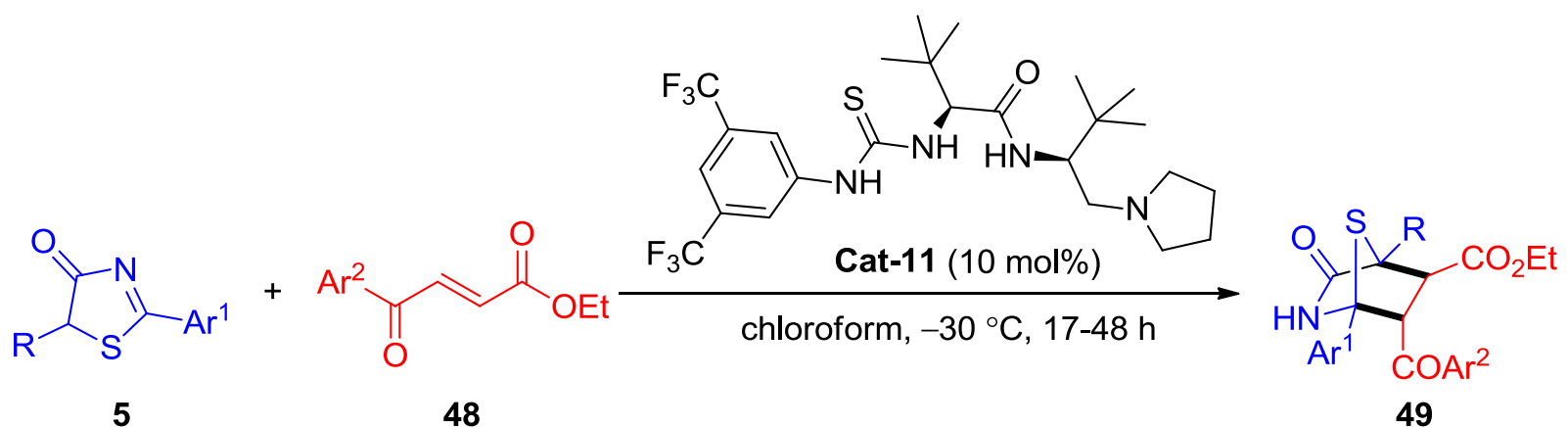

Scheme 23. Asymmetric [4+2] annulation reaction of thiazol-4(5H)-ones 5 with trans-4-oxo-4-arylbutenoates 48.

Table 13

\begin{tabular}{ccccccc}
\hline Entry & $\mathrm{R}$ & $\mathrm{Ar}^{1}$ & $\mathrm{Ar}^{2}$ & Yield (\%) & $\mathrm{dr}$ & ee (\%) \\
\hline 1 & $\mathrm{Me}$ & 2-quinolyl & $\mathrm{Ph}$ & 87 & $>20: 1$ & 95 \\
2 & $\mathrm{Me}$ & 2-quinolyl & $p-\mathrm{BrC}_{6} \mathrm{H}_{4}$ & 87 & $>20: 1$ & 97 \\
3 & $\mathrm{Me}$ & 2-quinolyl & 3,4- $\mathrm{Cl}_{2} \mathrm{C}_{6} \mathrm{H}_{3}$ & 96 & $>20: 1$ & 98 \\
4 & $\mathrm{Me}$ & 2-quinolyl & $p-\mathrm{MeOC}_{6} \mathrm{H}_{4}$ & 90 & $>20: 1$ & 98 \\
5 & $\mathrm{Me}$ & 2-quinolyl & 2-thienyl & 97 & $>20: 1$ & 99 \\
6 & $\mathrm{Me}$ & 2-quinolyl & 2-furyl & 96 & $>20: 1$ & 98 \\
7 & $\mathrm{Ph}$ & 2-quinolyl & $\mathrm{Ph}$ & 67 & $>20: 1$ & 90 \\
8 & $\mathrm{Me}$ & 2-pyridinyl & $\mathrm{Ph}$ & 95 & $>20: 1$ & 97 \\
\hline
\end{tabular}

Finally, the substrates scope of the reaction was elaborated using methyleneindolinones $\mathbf{5 0}$ (Scheme 24, Table 14). In this case, only one pair of diastereomers was obtained in high yields (up to 98\%) and perfect enantioselectivities (>99\%).

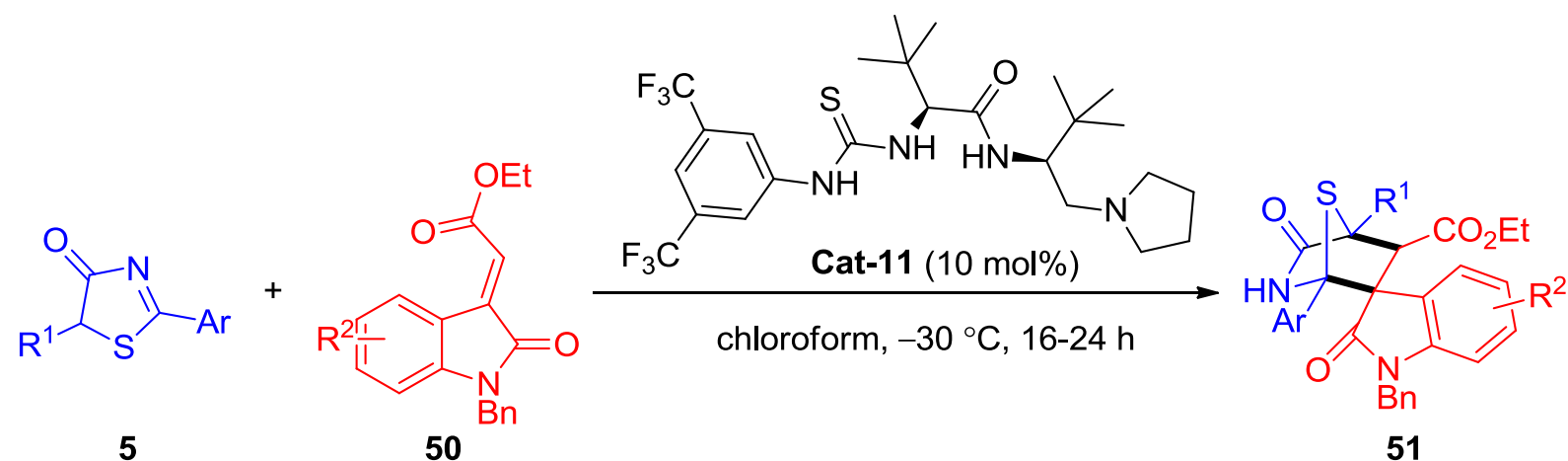

Scheme 24. Asymmetric [4+2] annulation reaction of thiazol-4(5H)-ones 5 with methyleneindolinones 50. 
Table 14

\begin{tabular}{ccccccc}
\hline Entry & $\mathrm{R}^{1}$ & $\mathrm{Ar}$ & $\mathrm{R}^{2}$ & Yield (\%) & $\mathrm{dr}$ & ee (\%) \\
\hline 1 & $\mathrm{Me}$ & 2-quinolyl & $\mathrm{H}$ & 96 & $>20: 1$ & 95 \\
2 & $\mathrm{Me}$ & 2-quinolyl & $5-\mathrm{F}$ & 98 & $>20: 1$ & 94 \\
3 & $\mathrm{Me}$ & 2-quinolyl & $5-\mathrm{Cl}$ & 97 & $>20: 1$ & 92 \\
4 & $\mathrm{Me}$ & 2-quinolyl & $5-\mathrm{Br}$ & 98 & $>20: 1$ & 90 \\
5 & $\mathrm{Me}$ & 2-quinolyl & $5-\mathrm{Me}$ & 95 & $>20: 1$ & $>99$ \\
6 & $\mathrm{Me}$ & 2-quinolyl & 5-OMe & 98 & $>20: 1$ & 98 \\
7 & $\mathrm{Ph}$ & 2-quinolyl & 6-Cl & 97 & $>20: 1$ & $>99$ \\
8 & $\mathrm{Me}$ & 2-quinolyl & 6-OMe & 94 & $>20: 1$ & $>99$ \\
9 & $\mathrm{Me}$ & 2-quinolyl & 7-Cl & 96 & $>20: 1$ & 93 \\
10 & $\mathrm{Me}$ & 2-quinolyl & 7-Me & 97 & $>20: 1$ & 96 \\
11 & $\mathrm{Me}$ & p-Cl-C $6 \mathrm{H}_{4}$ & $\mathrm{H}$ & 94 & $>20: 1$ & 90 \\
12 & $\mathrm{Me}$ & 2-pyridinyl & $\mathrm{H}$ & 96 & $>20: 1$ & 95 \\
13 & $\mathrm{Et}$ & 2-quinolyl & $\mathrm{H}$ & 87 & $>20: 1$ & $>99$ \\
14 & $\mathrm{Bn}$ & 2-quinolyl & $\mathrm{H}$ & 84 & $>20: 1$ & 97 \\
\hline
\end{tabular}

Stereoselective allylation of thiazol-4(5H)-ones 5 using a metallacyclic iridium complex Cat-12 in combination with a non-nucleophilic base $\mathrm{Mg}\left(\mathrm{N}^{i} \mathrm{Pr}_{2}\right)_{2}$ was reported by Hartwig and co-workers in 2014 (Scheme 25, Table 15). ${ }^{62}$ The reaction proceeded well through the formation of magnesium enolates to access enantioenriched allylation products which can readily be transformed to valuable tertiary thiols and thioethers with high yields (up to $96 \%$ ), stereoselectivities ( $d r$ up to $13: 1$ and ee up to $>99 \%$ ) with excellent regioselectivities (branched:linear $>20: 1$ ).
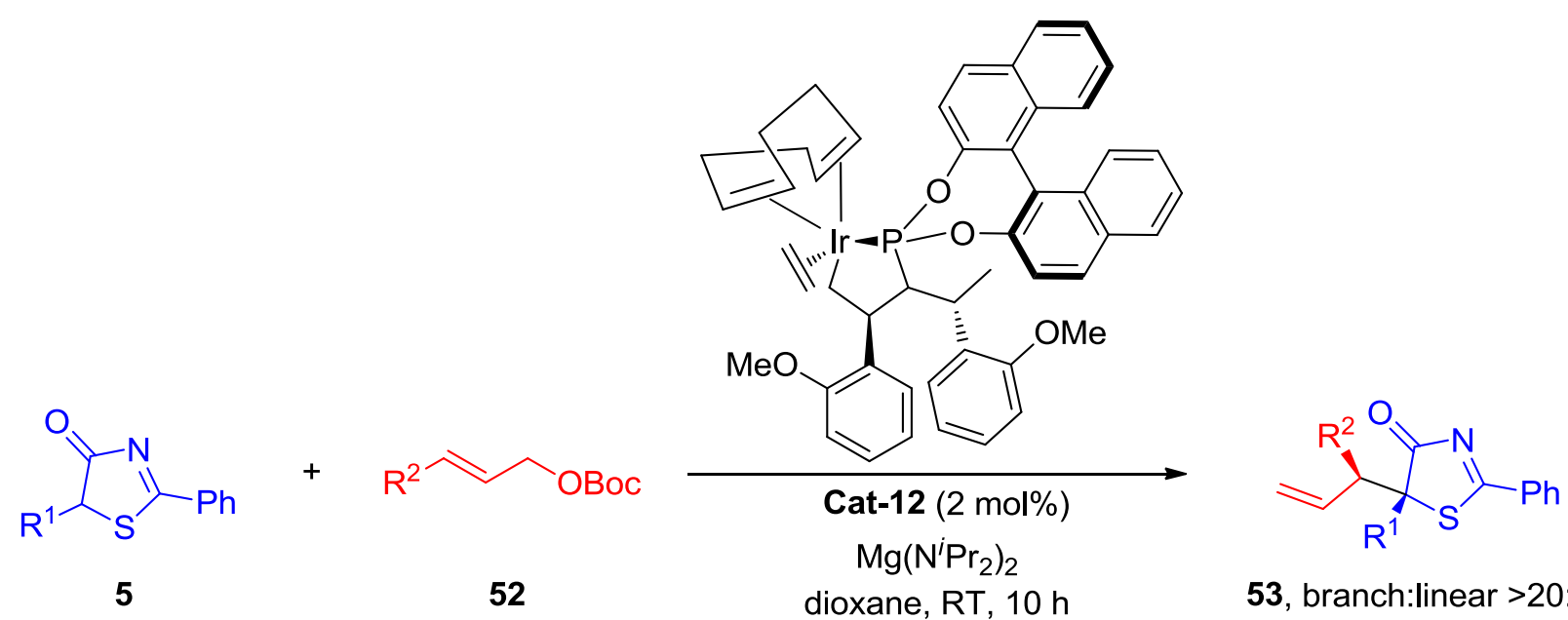

53, branch:linear $>20: 1$

Scheme 25. Asymmetric allylation of thiazol-4(5H)-ones 5.

In 2015, Lu and co-workers reported a modular pathway to access a wide range of chiral thiazolones containing tertiary chiral centers through the phosphine-catalyzed highly enantioselective $\gamma$-addition of thiazol-4(5H)-ones 5 to allenoate 54 (Scheme 26, Table 16). ${ }^{51}$ Several substituted thiazol-4(5H)-ones were 
easily converted to the corresponding chiral thiazolone derivatives $\mathbf{5 5}$ bearing sulfur heteroatom-containing tertiary chiral centers in high yields and excellent enantioselectivities (yield up to $97 \%$ and ee up to $95 \%$ ).

Table 15

\begin{tabular}{cccccc}
\hline Entry & $\mathrm{R}^{1}$ & $\mathrm{R}^{2}$ & Yield (\%) & $\mathrm{dr}$ & ee (\%) \\
\hline 1 & $\mathrm{Me}$ & $\mathrm{Ph}$ & 88 & 10.4 & 99 \\
2 & $\mathrm{Me}$ & $p-\mathrm{FC}_{6} \mathrm{H}_{4}$ & 92 & $10: 1$ & $>99$ \\
3 & $\mathrm{Me}$ & $p-\mathrm{ClC}_{6} \mathrm{H}_{4}$ & 94 & $10: 1$ & $>99$ \\
4 & $\mathrm{Me}$ & $m-\mathrm{BrC}_{6} \mathrm{H}_{4}$ & 92 & $12: 1$ & $>99$ \\
5 & $\mathrm{Me}$ & $p-\mathrm{CF}_{3} \mathrm{C}_{6} \mathrm{H}_{4}$ & 81 & $11: 1$ & $>99$ \\
6 & $\mathrm{Me}$ & $p-\mathrm{MeC}_{6} \mathrm{H}_{4}$ & 96 & $9: 1$ & 98 \\
7 & $\mathrm{Me}$ & $p-\mathrm{MeOC}_{6} \mathrm{H}_{4}$ & 85 & $10: 1$ & $>99$ \\
8 & $\mathrm{Me}$ & $2-\mathrm{naphthyl}$ & 84 & $8: 1$ & $>99$ \\
9 & $\mathrm{Me}$ & $3,4-\mathrm{Cl}{ }_{2} \mathrm{C}_{6} \mathrm{H}_{3}$ & 82 & $13: 1$ & $>99$ \\
10 & $\mathrm{Me}$ & $N-\mathrm{Boc}^{2}-\mathrm{indolyl}$ & 90 & $7: 1$ & 98 \\
11 & $\mathrm{Me}$ & $2-\mathrm{furyl}$ & 82 & $5: 1$ & $>99$ \\
12 & $\mathrm{Et}$ & $\mathrm{Ph}$ & 81 & $9: 1$ & 99 \\
13 & $\mathrm{Bn}$ & $\mathrm{Ph}$ & 79 & $4: 1$ & 98 \\
14 & $\mathrm{CH}_{2} \mathrm{CH}_{2} \mathrm{SCH}$ & $\mathrm{Ph}$ & 75 & $7: 1$ & $>99$ \\
\hline
\end{tabular}

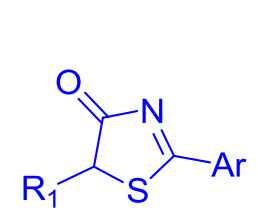

5

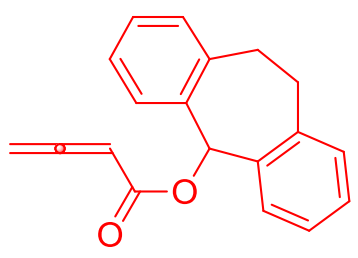

54

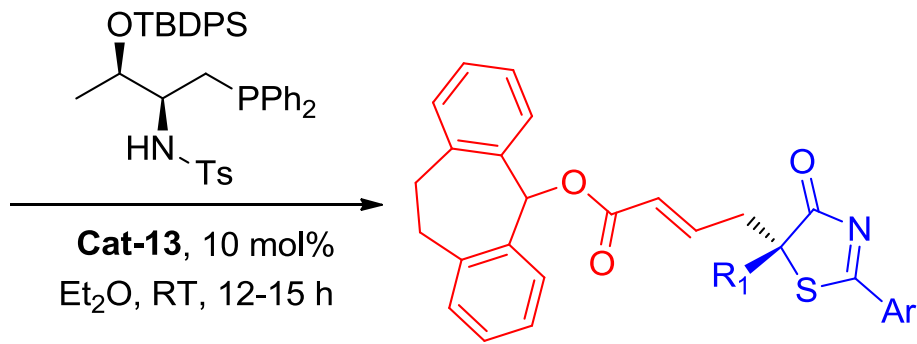

55

Scheme 26. Enantioselective $\gamma$-additions of thiazol-4(5H)-ones 5 to allenoate 54.

Table 16

\begin{tabular}{|c|c|c|c|c|c|c|c|c|c|}
\hline Entry & $\mathrm{R}^{1}$ & $\mathrm{Ar}$ & Yield (\%) & ee (\%) & Entry & $\mathrm{R}^{1}$ & $\mathrm{Ar}$ & Yield (\%) & ee $(\%)$ \\
\hline 1 & $\mathrm{Me}$ & $\mathrm{Ph}$ & 97 & 95 & 4 & ${ }^{i} \operatorname{Pr}$ & $\mathrm{Ph}$ & 92 & 92 \\
\hline 2 & Et & $\mathrm{Ph}$ & 95 & 94 & 5 & ${ }^{n} \mathrm{Bu}$ & $\mathrm{Ph}$ & 94 & 94 \\
\hline 3 & ${ }^{n} \mathrm{Pr}$ & $\mathrm{Ph}$ & 97 & 94 & 6 & ${ }^{i} \mathrm{Bu}$ & $\mathrm{Ph}$ & 89 & 88 \\
\hline 7 & $n-\mathrm{C}_{6} \mathrm{H}_{13}$ & $\mathrm{Ph}$ & 96 & 94 & 10 & $n-\mathrm{C}_{10} \mathrm{H}_{21}$ & $\mathrm{Ph}$ & 86 & 93 \\
\hline 8 & $\mathrm{CH}\left(\mathrm{CH}_{2}\right)_{5}$ & $\mathrm{Ph}$ & 95 & 93 & 11 & $\mathrm{Bn}$ & $\mathrm{Ph}$ & 90 & 92 \\
\hline 9 & $\left(\mathrm{CH}_{2}\right)_{2} \mathrm{SCH}_{3}$ & $\mathrm{Ph}$ & 93 & 90 & 12 & Me & 2-naphthyl & 96 & 89 \\
\hline
\end{tabular}


Interestingly, the thiazole substrate reacts with the alkynoate substrate $\mathbf{5 6}$ to afford the same product $\mathbf{5 5 a}$ with similar chemical yields and enantioselectivities (86\% yield and $95 \%$ ee) after $24 \mathrm{~h}$ (Scheme 26). The mechanism of the catalyst-substrate interactions and product formation were further investigated through DFT calculations.

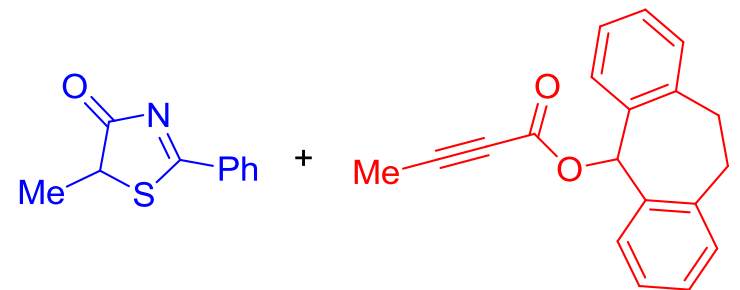

$5 \mathbf{a}$
56

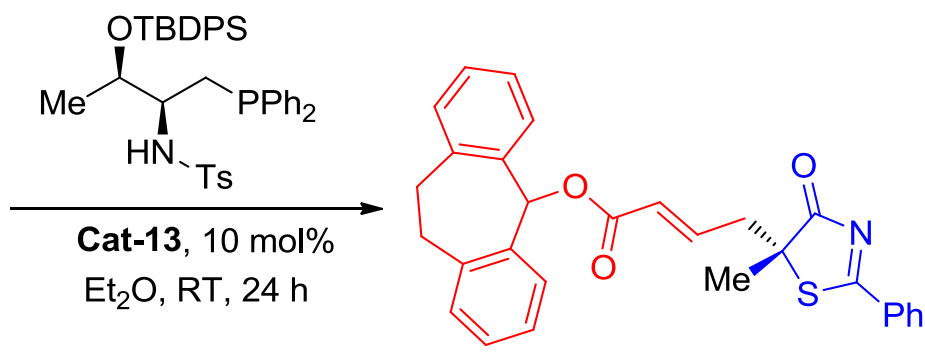

$55 a, 86 \%$ yield, $96 \%$ ee

Scheme 27. Enantioselective $\gamma$-additions of thiazol-4(5H)-one 5 a to alkynoate 56.

In 2018, Gong and co-workers developed the palladium-based enantioselective allylic C-H alkylation in combination with an optimized chiral phosphoramide ligand (Scheme 28, Table 17). ${ }^{63}$ When 1,4-pentadienes 57 were used in the enantioselective allylic $\mathrm{C}-\mathrm{H}$ alkylation of thiazol-4(5H)-ones $\mathbf{5}$, a competition of linear and branched products were observed (linear products are formed predominantly). A wide range of chiral substituted thiazol-4(5H)-one products (major product 58 ) bearing a quaternary stereogenic centre were obtained in high yields, regio- (linear products predominate over branched products) and stereoselectivities (dr up to $>20: 1$ and ee up to $93 \%$ ).

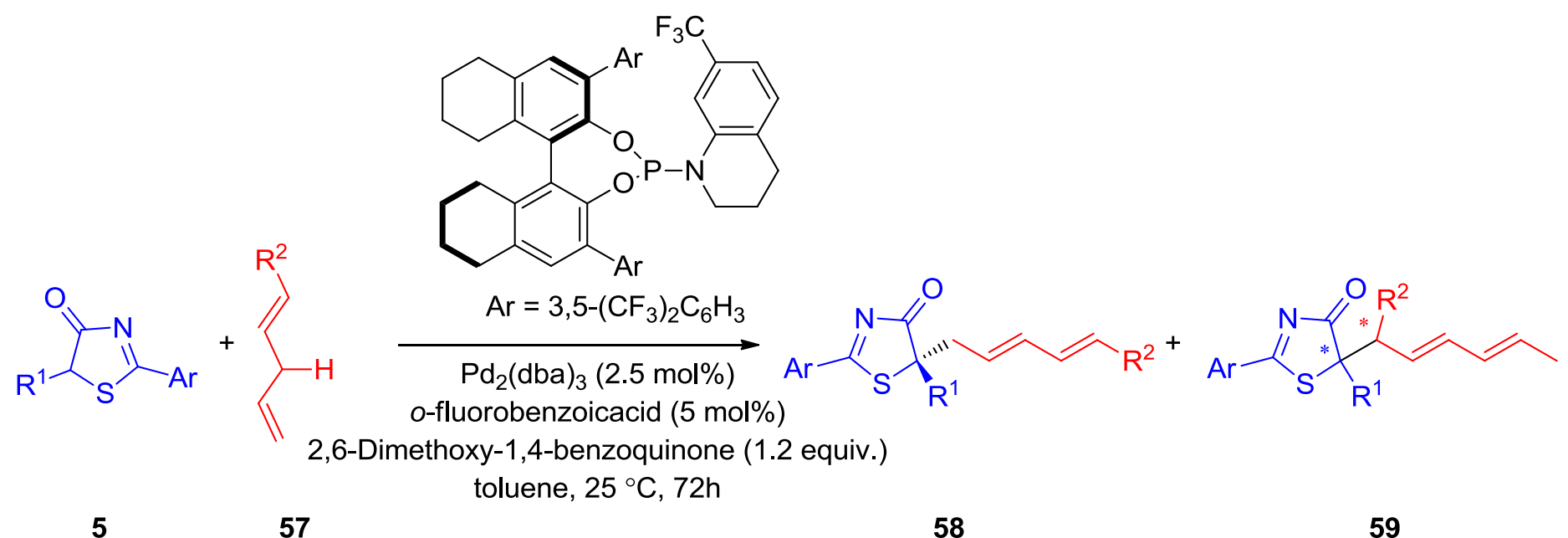

Scheme 28. Asymmetric allylic C-H alkylation of thiazol-4(5H)-ones 5 with 1,4-pentadienes 57 
Table 17

\begin{tabular}{|c|c|c|c|c|c|c|c|}
\hline Entry & $\mathrm{R}^{1}$ & $\mathrm{Ar}$ & $R^{2}$ & Yield (\%) & $58: 59$ & $\mathrm{dr}$ & ee (\%) \\
\hline 1 & $\mathrm{Bn}$ & $\mathrm{Ph}$ & $n-\mathrm{C}_{6} \mathrm{H}_{13}$ & 95 & $20: 1$ & $>20: 1$ & 90 \\
\hline 2 & $p-\mathrm{FC}_{6} \mathrm{H}_{4} \mathrm{CH}_{2}$ & $\mathrm{Ph}$ & $n-\mathrm{C}_{6} \mathrm{H}_{13}$ & 56 & $38: 1$ & $>20: 1$ & 56 \\
\hline 3 & $m-\mathrm{MeOC}_{6} \mathrm{H}_{4} \mathrm{CH}_{2}$ & $\mathrm{Ph}$ & $n-\mathrm{C}_{6} \mathrm{H}_{13}$ & 91 & $17: 1$ & $>20: 1$ & 84 \\
\hline 4 & $p-\mathrm{CNC}_{6} \mathrm{H}_{4} \mathrm{CH}_{2}$ & $\mathrm{Ph}$ & $n-\mathrm{C}_{6} \mathrm{H}_{13}$ & 93 & $23: 1$ & $>20: 1$ & 93 \\
\hline 5 & $2-\mathrm{C}_{10} \mathrm{H}_{7} \mathrm{CH}_{2}$ & $\mathrm{Ph}$ & $n-\mathrm{C}_{6} \mathrm{H}_{13}$ & 37 & $14: 1$ & $>20: 1$ & 90 \\
\hline 6 & $o-\mathrm{ClC}_{6} \mathrm{H}_{4} \mathrm{CH}_{2}$ & $\mathrm{Ph}$ & $n-\mathrm{C}_{6} \mathrm{H}_{13}$ & 89 & $13: 1$ & $>20: 1$ & 89 \\
\hline 7 & $m-\mathrm{ClC}_{6} \mathrm{H}_{4} \mathrm{CH}_{2}$ & $\mathrm{Ph}$ & $n-\mathrm{C}_{6} \mathrm{H}_{13}$ & 90 & $12: 1$ & $>20: 1$ & 91 \\
\hline 8 & $p-\mathrm{ClC}_{6} \mathrm{H}_{4} \mathrm{CH}_{2}$ & $\mathrm{Ph}$ & $n-\mathrm{C}_{6} \mathrm{H}_{13}$ & 87 & $12: 1$ & $>20: 1$ & 87 \\
\hline 9 & $m-\mathrm{MeC}_{6} \mathrm{H}_{4} \mathrm{CH}_{2}$ & $\mathrm{Ph}$ & $n-\mathrm{C}_{6} \mathrm{H}_{13}$ & 43 & $10: 1$ & $>20: 1$ & 85 \\
\hline 10 & $o-\mathrm{MeC}_{6} \mathrm{H}_{4} \mathrm{CH}_{2}$ & $\mathrm{Ph}$ & $n-\mathrm{C}_{6} \mathrm{H}_{13}$ & 52 & 19:1 & $>20: 1$ & 86 \\
\hline 11 & $p-\mathrm{MeC}_{6} \mathrm{H}_{4} \mathrm{CH}_{2}$ & $\mathrm{Ph}$ & $n-\mathrm{C}_{6} \mathrm{H}_{13}$ & 66 & 11:1 & $>20: 1$ & 87 \\
\hline 12 & $m-\mathrm{BrC}_{6} \mathrm{H}_{4} \mathrm{CH}_{2}$ & $\mathrm{Ph}$ & $n-\mathrm{C}_{6} \mathrm{H}_{13}$ & 89 & $15: 1$ & $>20: 1$ & 89 \\
\hline 13 & $o-\mathrm{BrC}_{6} \mathrm{H}_{4} \mathrm{CH}_{2}$ & $\mathrm{Ph}$ & $n-\mathrm{C}_{6} \mathrm{H}_{13}$ & 66 & $13: 1$ & $>20: 1$ & 88 \\
\hline 14 & $p-\mathrm{BrC}_{6} \mathrm{H}_{4} \mathrm{CH}_{2}$ & $\mathrm{Ph}$ & $n-\mathrm{C}_{6} \mathrm{H}_{13}$ & 92 & $20: 1$ & $>20: 1$ & 91 \\
\hline 15 & $\mathrm{H}$ & $\mathrm{Ph}$ & $n-\mathrm{C}_{6} \mathrm{H}_{13}$ & 94 & $30: 1$ & $>20: 1$ & 83 \\
\hline 16 & $p-\mathrm{NCC}_{6} \mathrm{H}_{4} \mathrm{CH}_{2}$ & $\mathrm{Ph}$ & Me & 95 & $20: 1$ & $>20: 1$ & 92 \\
\hline 17 & $p-\mathrm{NCC}_{6} \mathrm{H}_{4} \mathrm{CH}_{2}$ & $\mathrm{Ph}$ & $n-\mathrm{Pr}$ & 91 & $23: 1$ & $>20: 1$ & 91 \\
\hline 18 & $p-\mathrm{NCC}_{6} \mathrm{H}_{4} \mathrm{CH}_{2}$ & $\mathrm{Ph}$ & $n-\mathrm{C}_{9} \mathrm{H}_{17}$ & 90 & $38: 1$ & $>20: 1$ & 93 \\
\hline 18 & $p-\mathrm{NCC}_{6} \mathrm{H}_{4} \mathrm{CH}_{2}$ & $\mathrm{Ph}$ & $\mathrm{Ph}\left(\mathrm{CH}_{2}\right)_{2}$ & 91 & $20: 1$ & $>20: 1$ & 93 \\
\hline 19 & $p-\mathrm{NCC}_{6} \mathrm{H}_{4} \mathrm{CH}_{2}$ & $\mathrm{Ph}$ & $\mathrm{Cl}\left(\mathrm{CH}_{2}\right)_{2}$ & 81 & $21: 1$ & $>20: 1$ & 89 \\
\hline 20 & $p-\mathrm{NCC}_{6} \mathrm{H}_{4} \mathrm{CH}_{2}$ & $\mathrm{Ph}$ & cyclohexyl & 94 & $34: 1$ & $>20: 1$ & 83 \\
\hline 21 & $p-\mathrm{NCC}_{6} \mathrm{H}_{4} \mathrm{CH}_{2}$ & $\mathrm{Ph}$ & $\mathrm{Ph}$ & 81 & $24: 1$ & $>20: 1$ & 90 \\
\hline 22 & $p-\mathrm{NCC}_{6} \mathrm{H}_{4} \mathrm{CH}_{2}$ & $\mathrm{Ph}$ & $p-\mathrm{MeC}_{6} \mathrm{H}_{4}$ & 54 & $22: 1$ & $>20: 1$ & 91 \\
\hline .23 & $p-\mathrm{NCC}_{6} \mathrm{H}_{4} \mathrm{CH}_{2}$ & $\mathrm{Ph}$ & $p-\mathrm{MeOC}_{6} \mathrm{H}_{4}$ & 50 & $17: 1$ & $>20: 1$ & 92 \\
\hline 24 & $p-\mathrm{NCC}_{6} \mathrm{H}_{4} \mathrm{CH}_{2}$ & $\mathrm{Ph}$ & $m-\mathrm{ClC}_{6} \mathrm{H}_{4}$ & 86 & $40: 1$ & $>20: 1$ & 93 \\
\hline 25 & $p-\mathrm{NCC}_{6} \mathrm{H}_{4} \mathrm{CH}_{2}$ & $\mathrm{Ph}$ & $\mathrm{H}$ & 73 & $5: 1$ & $>20: 1$ & 84 \\
\hline 26 & $p-\mathrm{NCCC}_{6} \mathrm{H}_{4} \mathrm{CH}_{2}$ & $\mathrm{Ph}$ & $\mathrm{EtO}_{2} \mathrm{C}\left(\mathrm{CH}_{2}\right)_{2}$ & 99 & $11: 1$ & $>20: 1$ & 84 \\
\hline 27 & $p-\mathrm{NCCC}_{6} \mathrm{H}_{4} \mathrm{CH}_{2}$ & $\mathrm{Ph}$ & $\mathrm{BnNHCO}\left(\mathrm{CH}_{2}\right)_{2}$ & 79 & $10: 1$ & $>20: 1$ & 83 \\
\hline 28 & $p-\mathrm{NCC}_{6} \mathrm{H}_{4} \mathrm{CH}_{2}$ & $\mathrm{Ph}$ & $\mathrm{TsO}\left(\mathrm{CH}_{2}\right)_{2}$ & 92 & $16: 1$ & $>20: 1$ & 90 \\
\hline 29 & $\mathrm{Ph}$ & $\mathrm{Ph}$ & Me & 90 & $11: 1$ & $5: 1$ & 87 \\
\hline 30 & $\mathrm{Ph}$ & $m-\mathrm{MeC}_{6} \mathrm{H}_{4}$ & $\mathrm{Me}$ & 70 & $10: 1$ & $6: 1$ & 91 \\
\hline 31 & $\mathrm{Ph}$ & $p-\mathrm{BrC}_{6} \mathrm{H}_{4}$ & Me & 87 & $13: 1$ & $4: 1$ & 88 \\
\hline 32 & $\mathrm{Ph}$ & $p-\mathrm{FC}_{6} \mathrm{H}_{4}$ & Me & 80 & $10: 1$ & $5: 1$ & 88 \\
\hline 33 & $\mathrm{Ph}$ & $\mathrm{Ph}$ & $\mathrm{H}$ & 92 & - & $13: 1$ & 89 \\
\hline 34 & $\mathrm{Ph}$ & $m-\mathrm{MeC}_{6} \mathrm{H}_{4}$ & $n$-Bu & 57 & 10:1 & $3: 1$ & 91 \\
\hline 35 & $\mathrm{Ph}$ & $m-\mathrm{MeC}_{6} \mathrm{H}_{4}$ & $n-\operatorname{Pr}$ & 75 & $10: 1$ & $9: 1$ & 91 \\
\hline
\end{tabular}


Stereoselective 1,4-conjugate addition process of thiazol-4(5H)-ones 5 with $\mathrm{N}$-maleimides 60 or 1,4 naphthoquinones 62 were developed by Jiang and co-workers in 2016 based on a dipeptide-derived tertiary amine catalyst Cat-14. ${ }^{64}$

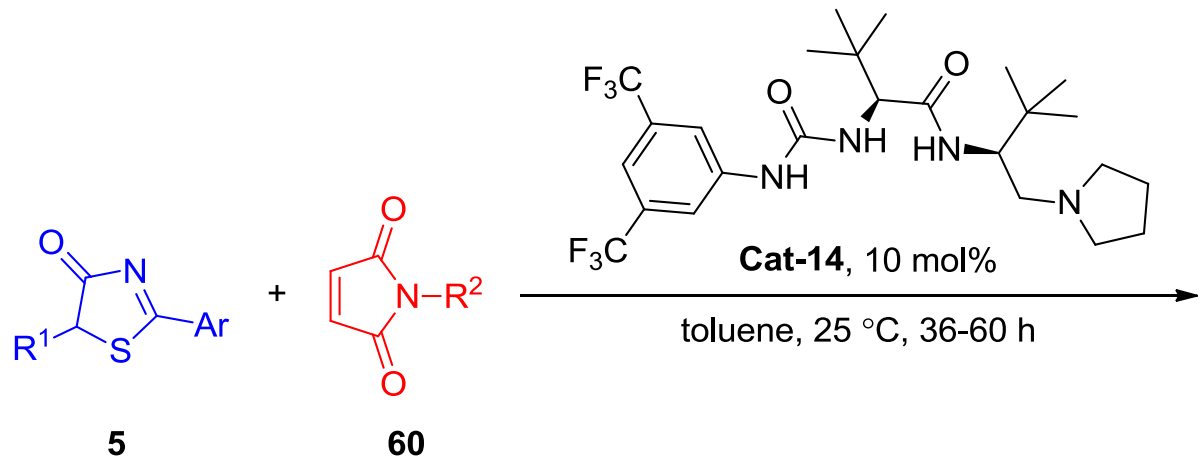

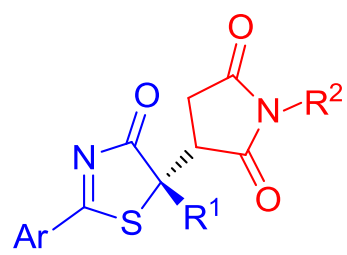

61

Scheme 29. Asymmetric 1,4-conjugate addition of thiazol-4(5H)-ones 5 with maleimides 60.

A series of valuable heterocyclic compounds 61 and 63 , derived from the $N$-maleimides 60 or $1,4-$ naphthoquinones 62 were obtained with excellent enantio-/disastereo-selectivities (up to 99\% ee, dr >20:1) and yields (up to 97\%) (Scheme 29, Table 18 \& Scheme 30). The absolute configuration of one substrate was determined trough X-ray crystallographic analysis, and the configuration of other substrates of 61 were determined by analogy.

Table 18

\begin{tabular}{|c|c|c|c|c|c|c|}
\hline Entry & $\mathrm{R}^{1}$ & $\mathrm{Ar}$ & $\mathrm{R}^{2}$ & Yield (\%) & $\mathrm{dr}$ & ee $(\%)$ \\
\hline 1 & $\mathrm{Me}$ & $\mathrm{Ph}$ & $\mathrm{Ph}$ & 96 & $>20: 1$ & 97 \\
\hline 2 & $\mathrm{Me}$ & $\mathrm{Ph}$ & $p-\mathrm{FC}_{6} \mathrm{H}_{4}$ & 97 & $>20: 1$ & 97 \\
\hline 3 & $\mathrm{Me}$ & $\mathrm{Ph}$ & $p-\mathrm{ClC}_{6} \mathrm{H}_{4}$ & 89 & $>20: 1$ & 98 \\
\hline 4 & $\mathrm{Me}$ & $\mathrm{Ph}$ & $m-\mathrm{BrC}_{6} \mathrm{H}_{4}$ & 95 & $>20: 1$ & 99 \\
\hline 5 & $\mathrm{Me}$ & $\mathrm{Ph}$ & $3,5-\mathrm{F}_{2} \mathrm{C}_{6} \mathrm{H}_{3}$ & 92 & $>20: 1$ & 98 \\
\hline 6 & $\mathrm{Me}$ & $\mathrm{Ph}$ & $m-\mathrm{ClC}_{6} \mathrm{H}_{4}$ & 93 & $>20: 1$ & 98 \\
\hline 7 & $\mathrm{Me}$ & $\mathrm{Ph}$ & $m-\mathrm{BrC}_{6} \mathrm{H}_{4}$ & 97 & $>20: 1$ & 97 \\
\hline 8 & $\mathrm{Me}$ & $\mathrm{Ph}$ & $p-\mathrm{MeC}_{6} \mathrm{H}_{4}$ & 96 & $>20: 1$ & 98 \\
\hline 9 & $\mathrm{Me}$ & $\mathrm{Ph}$ & $m-\mathrm{MeC}_{6} \mathrm{H}_{4}$ & 95 & $>20: 1$ & 99 \\
\hline 10 & $\mathrm{Me}$ & $\mathrm{Ph}$ & $o-\mathrm{MeC}_{6} \mathrm{H}_{4}$ & 92 & $>20: 1$ & 97 \\
\hline 11 & $\mathrm{Me}$ & $\mathrm{Ph}$ & $p-\mathrm{MeOC}_{6} \mathrm{H}_{4}$ & 93 & $>20: 1$ & 98 \\
\hline 12 & $\mathrm{Me}$ & $\mathrm{Ph}$ & $m-\mathrm{MeOC}_{6} \mathrm{H}_{4}$ & 84 & $>20: 1$ & 94 \\
\hline 13 & $\mathrm{Me}$ & $\mathrm{Ph}$ & $\mathrm{Bn}$ & 90 & $>20: 1$ & 98 \\
\hline 14 & $\mathrm{Me}$ & $\mathrm{Ph}$ & Me & 91 & $>20: 1$ & 97 \\
\hline 15 & Et & $\mathrm{Ph}$ & $\mathrm{Ph}$ & 87 & $>20: 1$ & 97 \\
\hline 16 & ${ }^{i} \operatorname{Pr}$ & $\mathrm{Ph}$ & $\mathrm{Ph}$ & 93 & $>20: 1$ & 95 \\
\hline 17 & $\mathrm{Bn}$ & $\mathrm{Ph}$ & $\mathrm{Ph}$ & 92 & $>20: 1$ & 97 \\
\hline 18 & $\mathrm{Me}$ & 2-quinolyl & $\mathrm{Ph}$ & 82 & $>20: 1$ & 96 \\
\hline
\end{tabular}




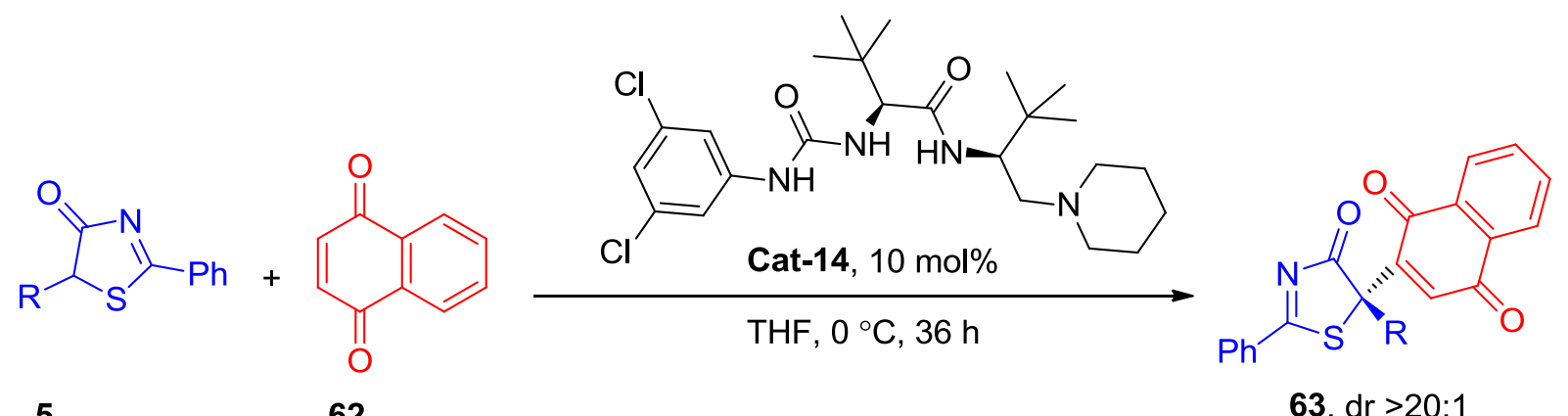

5

62

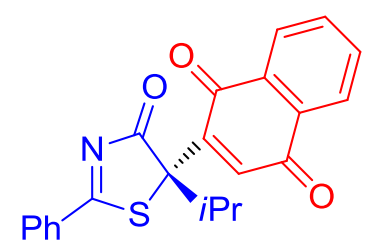

Yield: $71 \%$, ee: $90 \%$

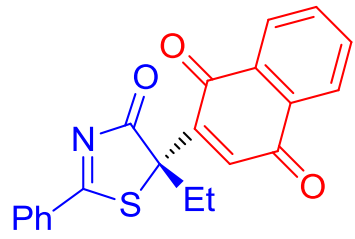

Yield: $70 \%$, ee: $91 \%$

Yield: $71 \%$, ee: $70 \%$

Scheme 30. Asymmetric 1,4-conjugate addition of thiazol-4(5H)-ones 5 with 1,4-naphthoquinones 62.

In 2018, Li and co-workers reported diastereodivergent asymmetric reactions of thiazol-4(5H)-ones 5 with $p$-quinone methides and $o$-quinone methides catalyzed by two pseudoenantiomeric catalysts. ${ }^{65} \mathrm{~A}$ wide range of adducts possessing vicinal sulfur-functionalized quaternary and tertiary stereocenters were obtained in high yields with excellent stereoselectivities (Schemes 31-33).<smiles>[R]C1SC([Al])=NC1=O</smiles>

5<smiles>[R17]c1ccc(/C=C2\C=C(CCC)C(=O)C(C(C)(C)C)=C2)c(O)c1</smiles>

64

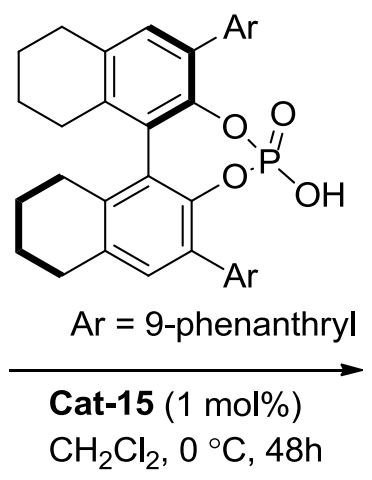

$\mathrm{CH}_{2} \mathrm{Cl}_{2}, 0^{\circ} \mathrm{C}, 48 \mathrm{~h}$

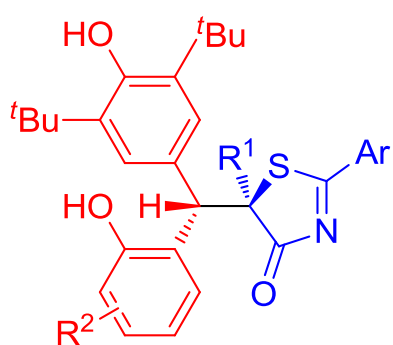

65

Scheme 31. Asymmetric reactions of thiazol-4(5H)-ones 5 with $p$-quinone methides 64 .

The reaction of thiazol-4(5H)-ones 5 with $p$-quinone methides 64 were first carried out in presence of a naphthol-based phosphoric acid catalyst Cat-15 to obtain the substituted thiazol-4(5H)-ones adducts 65 with very high yield (up to $97 \%$ ), enantioselectivity (ee up to $>99 \%$ ) and excellent diastereoselctivity (dr $>20: 1$ ) (Scheme 31, Table 19). 
Table 19

\begin{tabular}{ccccccc}
\hline Entry & $\mathrm{R}^{1}$ & $\mathrm{Ar}$ & $\mathrm{R}^{2}$ & Yield (\%) & $\mathrm{dr}$ & ee (\%) \\
\hline 1 & $\mathrm{Me}$ & $\mathrm{Ph}$ & $\mathrm{H}$ & 94 & $>20: 1$ & 96 \\
2 & $\mathrm{Me}$ & $\mathrm{Ph}$ & $4-\mathrm{F}$ & 90 & $>20: 1$ & 90 \\
3 & $\mathrm{Me}$ & $\mathrm{Ph}$ & $4-\mathrm{Cl}$ & 89 & $>20: 1$ & 86 \\
4 & $\mathrm{Me}$ & $\mathrm{Ph}$ & $4-\mathrm{Me}$ & 83 & $>20: 1$ & 92 \\
5 & $\mathrm{Me}$ & $\mathrm{Ph}$ & $5-\mathrm{Br}$ & 93 & $>20: 1$ & 96 \\
6 & $\mathrm{Me}$ & $\mathrm{Ph}$ & $6-\mathrm{MeO}$ & 85 & $>20: 1$ & 94 \\
7 & $\mathrm{Me}$ & $p-\mathrm{FC}_{6} \mathrm{H}_{4}$ & $\mathrm{H}$ & 95 & $>20: 1$ & 94 \\
8 & $\mathrm{Me}$ & $p-\mathrm{ClC}_{6} \mathrm{H}_{4}$ & $\mathrm{H}$ & 97 & $>20: 1$ & 92 \\
9 & $\mathrm{Me}$ & $p-\mathrm{MeC}_{6} \mathrm{H}_{4}$ & $\mathrm{H}$ & 93 & $>20: 1$ & 90 \\
10 & $\mathrm{Me}$ & $m$ - $\mathrm{FC}_{6} \mathrm{H}_{4}$ & $\mathrm{H}$ & 94 & $>20: 1$ & 96 \\
11 & $\mathrm{Me}$ & $m$ - $\mathrm{BrC}_{6} \mathrm{H}_{4}$ & $\mathrm{H}$ & 93 & $>20: 1$ & 96 \\
12 & $\mathrm{Me}$ & $m$ - $\mathrm{MeOC}_{6} \mathrm{H}_{4}$ & $\mathrm{H}$ & 94 & $>20: 1$ & 94 \\
13 & $\mathrm{Me}$ & o- $\mathrm{FC}_{6} \mathrm{H}_{4}$ & $\mathrm{H}$ & 84 & $>20: 1$ & 96 \\
14 & $\mathrm{Me}$ & 2-thienyl & $\mathrm{H}$ & 93 & $>20: 1$ & 80 \\
15 & $\mathrm{Me}$ & 2-pyridinyl & $\mathrm{H}$ & 94 & $>20: 1$ & 98 \\
16 & $\mathrm{Et}$ & 2-pyridinyl & $\mathrm{H}$ & 88 & $>20: 1$ & 94 \\
17 & ${ }^{n} \mathrm{Pr}$ & 2-pyridinyl & $\mathrm{H}$ & 85 & $>20: 1$ & 88 \\
18 & $\mathrm{Me}$ & 3-pyridinyl & $\mathrm{H}$ & 85 & $>20: 1$ & $>99$ \\
19 & $\mathrm{Bn}$ & $\mathrm{Ph}^{2}$ & $\mathrm{H}$ & 83 & $>20: 1$ & 82 \\
\hline
\end{tabular}

However, when the same reactions were performed in the presence of a squaramide catalyst Cat-16, the diastereoisomers of the products 65, i.e. 66, were obtained (Scheme 32, Table 20) with high yields and stereoselectivities (yield up to $99 \%$, dr up to $>20: 1$, ee up to $99 \%$ for 66 ).

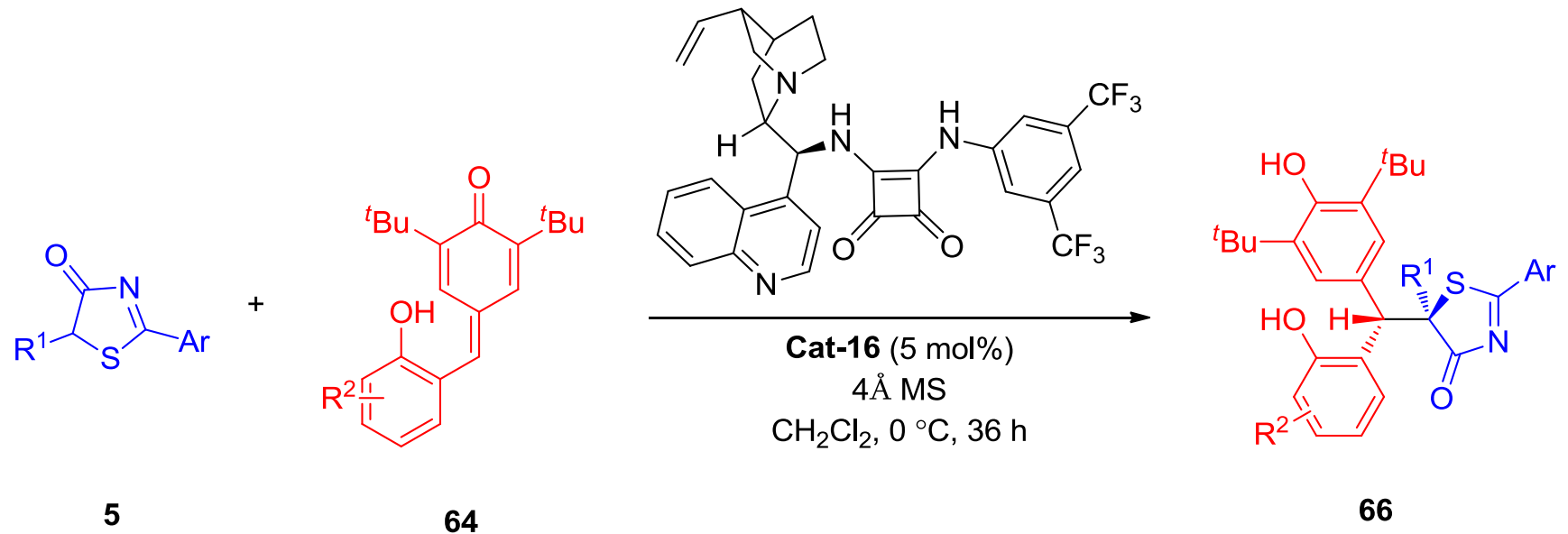

Scheme 32. Asymmetric reactions of $p$-quinone methides 64 with thiazol-4(5H)-ones 5. 
Table 20

\begin{tabular}{ccccccc}
\hline Entry & $\mathrm{R}^{1}$ & $\mathrm{Ar}$ & $\mathrm{R}^{2}$ & Yield (\%) & $\mathrm{dr}$ & ee (\%) \\
\hline 1 & $\mathrm{Me}$ & $\mathrm{Ph}$ & $\mathrm{H}$ & 83 & $15: 1$ & 92 \\
2 & $\mathrm{Me}$ & $\mathrm{Ph}$ & 5-OMe & 98 & $>20: 1$ & 88 \\
3 & $\mathrm{Me}$ & $\mathrm{Ph}$ & $4-\mathrm{Me}$ & 84 & $>20: 1$ & 92 \\
4 & $\mathrm{Me}$ & $\mathrm{Ph}$ & $4-\mathrm{Br}$ & 76 & $>20: 1$ & 96 \\
5 & $\mathrm{Me}$ & $\mathrm{Ph}$ & $6-\mathrm{MeO}$ & 93 & $>20: 1$ & 99 \\
6 & $\mathrm{Bn}$ & $\mathrm{Ph}$ & $\mathrm{H}$ & 87 & $6: 1$ & 78 \\
7 & $\mathrm{Me}$ & $p-\mathrm{FC}_{6} \mathrm{H}_{4}$ & $\mathrm{H}$ & 81 & $>20: 1$ & 92 \\
8 & $\mathrm{Me}$ & $p$ - $\mathrm{ClC}_{6} \mathrm{H}_{4}$ & $\mathrm{H}$ & 85 & $11: 1$ & 98 \\
9 & $\mathrm{Me}$ & $p$ - $\mathrm{MeC}_{6} \mathrm{H}_{4}$ & $\mathrm{H}$ & 88 & $13: 1$ & 94 \\
10 & $\mathrm{Me}$ & o- $\mathrm{FC}_{6} \mathrm{H}_{4}$ & $\mathrm{H}$ & 87 & $15: 1$ & 86 \\
11 & $\mathrm{Me}$ & - $\mathrm{BrC}_{6} \mathrm{H}_{4}$ & $\mathrm{H}$ & 86 & $>20: 1$ & 88 \\
12 & $\mathrm{Me}$ & 2-thienyl & $\mathrm{H}$ & 86 & $11: 1$ & 86 \\
13 & $\mathrm{Me}$ & 2-pyridinyl & $\mathrm{H}$ & 99 & $>20: 1$ & 98 \\
14 & $\mathrm{Et}$ & 2-pyridinyl & $\mathrm{H}$ & 83 & $18: 1$ & 92 \\
15 & $\mathrm{Me}$ & 3-pyridinyl & $\mathrm{H}$ & 86 & $12: 1$ & 88 \\
\hline
\end{tabular}

Next, the method was applied to the reaction of substituted thiazol-4(5H)-ones 5 with o-hydroxybenzyl alcohols 67 in presence of a binaphthol-based phosphoric acid catalyst. The reaction proceeded through the 0 quinone methides intermediates (Scheme 33, Table 21). The adducts 68 were obtained in high yields and stereoselectivities (yield up to $95 \%$, dr up to $>20: 1$, ee up to $99 \%$ ).

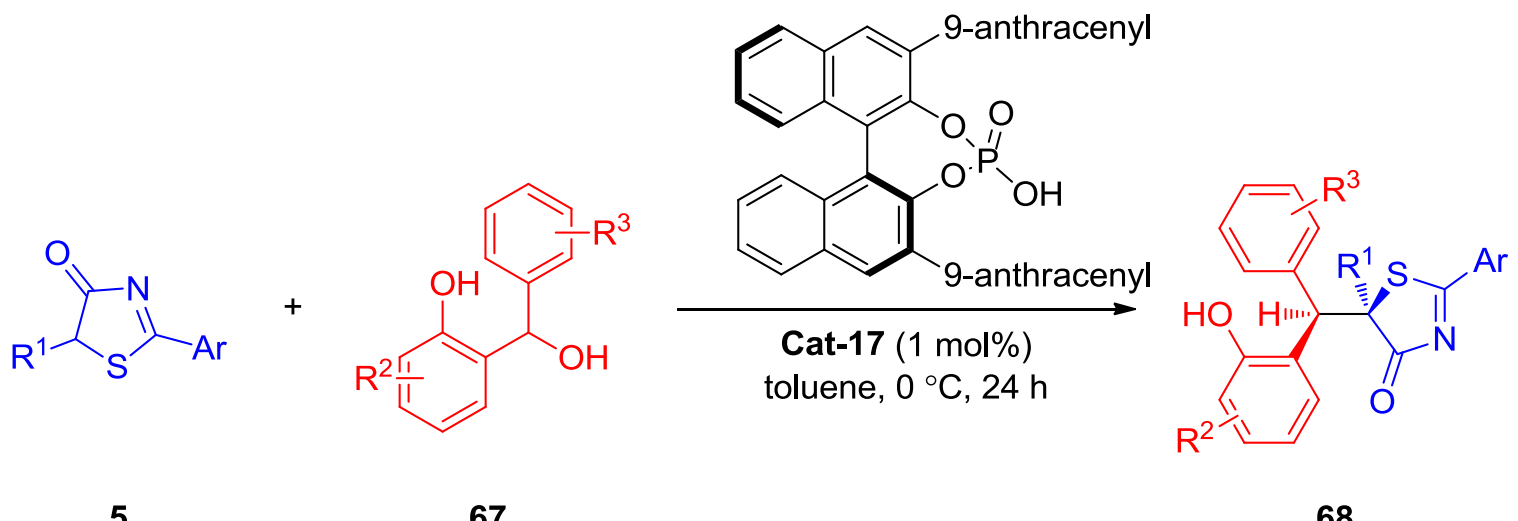

5

67

68

Scheme 33. Asymmetric reactions of $o$-hydroxybenzyl alcohols 67 with thiazol-4(5H)-ones 5. 
Table 21

\begin{tabular}{cccccccc}
\hline Entry & $\mathrm{R}^{1}$ & $\mathrm{Ar}$ & $\mathrm{R}^{2}$ & $\mathrm{R}^{3}$ & Yield (\%) & $\mathrm{dr}$ & ee (\%) \\
\hline 1 & $\mathrm{Me}$ & $\mathrm{Ph}$ & $\mathrm{H}$ & $4-\mathrm{MeO}$ & 87 & $19: 1$ & 98 \\
2 & $\mathrm{Me}$ & $\mathrm{Ph}$ & $4-\mathrm{Br}$ & $4-\mathrm{MeO}$ & 81 & $10: 1$ & 89 \\
3 & $\mathrm{Me}$ & $\mathrm{Ph}$ & $4-\mathrm{Me}$ & $4-\mathrm{MeO}$ & 87 & $13: 1$ & 94 \\
4 & $\mathrm{Me}$ & $\mathrm{Ph}$ & $\mathrm{H}$ & $\mathrm{H}$ & 95 & $>20: 1$ & 96 \\
5 & $\mathrm{Me}$ & $\mathrm{Ph}$ & $\mathrm{H}$ & $4-\mathrm{F}$ & 84 & $13: 1$ & 86 \\
6 & $\mathrm{Me}$ & $\mathrm{Ph}$ & $\mathrm{H}$ & $4-\mathrm{Cl}$ & 94 & $17: 1$ & 94 \\
7 & $\mathrm{Me}$ & $\mathrm{Ph}$ & $\mathrm{H}$ & $3-\mathrm{Me}$ & 84 & $18: 1$ & 92 \\
8 & $\mathrm{Me}$ & $\mathrm{Ph}$ & $\mathrm{H}$ & $2-\mathrm{Me}$ & 82 & $17: 1$ & 94 \\
9 & $\mathrm{Me}$ & $p-\mathrm{FC}_{6} \mathrm{H}_{4}$ & $\mathrm{H}$ & $4-\mathrm{MeO}$ & 84 & $13: 1$ & 95 \\
10 & $\mathrm{Me}$ & $p-\mathrm{ClC}_{6} \mathrm{H}_{4}$ & $\mathrm{H}$ & $4-\mathrm{MeO}$ & 89 & $>20: 1$ & 99 \\
11 & $\mathrm{Me}$ & $m$ - $\mathrm{BrC}_{6} \mathrm{H}_{4}$ & $\mathrm{H}$ & $4-\mathrm{MeO}$ & 86 & $>20: 1$ & 96 \\
12 & $\mathrm{Me}$ & $m-\mathrm{MeOC}_{6} \mathrm{H}_{4}$ & $\mathrm{H}$ & $4-\mathrm{MeO}$ & 83 & $14: 1$ & 95 \\
13 & $\mathrm{Me}$ & $0-\mathrm{FC}_{6} \mathrm{H}_{4}$ & $\mathrm{H}$ & $4-\mathrm{MeO}$ & 90 & $16: 1$ & 86 \\
14 & $\mathrm{Me}$ & $2-\mathrm{pyridinyl}_{10}$ & $\mathrm{H}$ & $4-\mathrm{MeO}$ & 76 & $15: 1$ & 92 \\
15 & $\mathrm{Bn}$ & $\mathrm{Ph}$ & $\mathrm{H}$ & $4-\mathrm{MeO}$ & 88 & $9: 1$ & 80 \\
\hline
\end{tabular}

\section{Conclusions}

Substituted chiral rhodanines, thiazolidine-2,4-diones and thiazol-4(5H)-ones are the growing group of chiral compounds. Catalysis has made enormous strides in the last few years on asymmetric reactions of rhodanines, thiazolidine-2,4-diones and thiazol-4(5H)-ones, which in turn can further enlighten the design of new reactions using these nucleophile substrates. In this review, we have shown the development of catalytic enantioselective methods using rhodanine, thiazolidine-2,4-dione and thiazol-4(5H)-one as nucleophile to synthesize a wide range of chiral derivatives including synthetic procedures used to access the nucleophiles themselves. There are still several challenges, such as catalytic asymmetric reactions of fluorinated representatives of these classes, or reactions in aqueous media for further derivatization, which need to be addressed.

\section{Acknowledgements}

Help with literature searching and commenting on manuscript by Subhdeep Paladhi, IIT Kharagpur, India is gratefully acknowledged. We sincerely thank the editors and reviewers for their invaluable comments and suggestions.

\section{References}

1. Brown, C. F. Chem. Rev. 1961, 61, 463-521. 
http://dx.doi.org/10.1021/cr60213a002

2. Singh, S. P.; Parmar, S. S.; Raman, K.; Steinberg, V. I. Chem. Rev. 1981, 81, 175-203. http://dx.doi.org/10.1021/cr00042a003

3. Lesyk, R. B.; Zimenkovsky, B. S. Curr. Org. Chem. 2004, 8, 1547-1577. https://doi.org/10.2174/1385272043369773

4. Grummt, U. V.; Weiss, D.; Birckner, E.; Beckert, R. J. Phys. Chem. A 2007, 111, 1104- 1110. http://dx.doi.org/10.1021/jp0672003

5. Sortino, M.; Delgado, P.; Juárez, S.; Quiroga, J.; Abonía, R.; Insuasty, B.; Nogueras, M.; Rodero, L.; Garibotto, F. M.; Enriz, R. D.; Zacchino, S. A. Bioorg. Med. Chem. 2007, 15, 484-494. https://doi.org/10.1016/i.bmc.2006.09.038

6. Murugan, R.; Anbazhagan, S.; Narayanan, S. S. Eur. J. Med. Chem. 2009, 44, 3272-3279. https://doi.org/10.1016/i.ejmech.2009.03.035

7. Tomasič, T.; Masič, L. P. Curr. Med. Chem. 2009, 16, 1596-1629. http://dx.doi.org/10.2174/092986709788186200

8. Véniant, M. M.; Hale, C.; Hungate, R. W.; Gahm, K.; Emery, M. G.; Jona, J.; Joseph, S.; Adams,J.; Hague, A.; Moniz, G.; Zhang, J.; Bartberger, M. D.; Li, V.; Syed, R.; Jordan, S.; Komorowski, R.; Chen, M. M.; Cupples, R.; Kim, K. W.; St Jean, D. J.; Johansson, L.; Henriksson, M. A.; Williams, M.; Vallgårda, J.; Fotsch, C.;

Wang, M. J. J. Med. Chem. 2010, 53, 4481-4487.

http://dx.doi.org/10.1021/jm100242d

9. Tomašić, T.; Zidar, N.; Mueller-Premru, M.; Kikelj, D.; Mašić, L. P. Eur. J. Med. Chem. 2010, 45, $1667-1672$.

10. https://doi.org/10.1016/j.ejmech.2009.12.030

11. Tomašić, T.; Zidar, N.; Kovać, A.; Turk, S.; Simćić, M.; Blanot, D.; Mueller-Premru, M.; Filipić, M.; Grdadolnik, S. G.; Zega, A.; Anderluh, M.; Gobec, S.; Kikelj, D.; Mašić, L. P. ChemMedChem, 2010, 5, 286295.

12. https://doi.org/10.1002/cmdc.200900449

13. Ono, M.; Hayashi, S.; Matsumura, K.; Kimura, H.; Okamoto, Y.; Ihara, M.; Takahashi, R.; Mori, H.; Saji, H. ACS Chem. Neurosci. 2011, 2, 269-275.

http://dx.doi.org/10.1021/cn200002t

14. Heng, S.; Tieu, W.; Hautmann, S.; Kuan, K.; Sejer Pedersen, D.; Pietsch, M.; Gütschow, M.; Abell, A. D. Bioorg. Med. Chem. 2011, 19, 7453-7463.

https://doi.org/10.1016/i.bmc.2011.10.042

15. Ma, L. A.; Xie, C. F.; Ma, Y. H.; Liu, J. A.; Xiang, M. L.; Ye, X.; Zheng, H.; Chen, Z. Z.; Xu, Q. Y.; Chen, T.; Chen, J. Y.; Yang, J. C.; Qiu, N.; Wang, G. C.; Liang, X. L.; Peng, A. H.; Yang, S. Y.; Wei, Y. Q.; Chen, L. J. J. Med. Chem. 2011, 54, 2060-2068. http://dx.doi.org/10.1021/jm1011534

16. Mendgen, T.; Steuer, C.; Klein, C. D. J. Med. Chem. 2012, 55, 743-753. http://dx.doi.org/10.1021/jm201243p

17. Jain, A. K.; Vaidya, A.; Ravichandran, V.; Kashaw, S. K.; Agrawal, R. K. Bioorg. Med. Chem. 2012, 20, 33783395.

https://doi.org/10.1016/i.bmc.2012.03.069

18. Jain, V. S.; Vora, D. K.; Ramaa, C. S. Bioorg. Med. Chem. 2013, 21, 1599-1620. https://doi.org/10.1016/i.bmc.2013.01.029

19. Khalil, N. A.; Ahmed, E. M.; El-Nassan, H. B. Med. Chem. Res. 2013, 22, 1021-1027. https://doi.org/10.1007/s00044-012-0098-7 
20. Bhatti, R. S.; Shah, S.; Suresh, P.; Krishan, P.; Sandhu, J. S. Int. J. Med. Chem. 2013, 2013, 1-16. http://dx.doi.org/10.1155/2013/793260

21. Desai, N. C.; Pandit, U. P.; Dodiya, A. Expert Opin. Ther. Pat. 2015, 25, 479-488. https://doi.org/10.1517/13543776.2014.1001738

22. Naim, M. J.; Alam, M. J.; Ahmad, S.; Nawaz, F.; Shrivastava, N.; Sahu, M.; Alam, O. Eur. J. Med. Chem. 2017, 129, 218-250.

https://doi.org/10.1016/i.ejmech.2017.02.031

23. Kaminskyy, D.; Kryshchyshyn, A.; Lesyk, R. Eur. J. Med. Chem. 2017, 140, 542-594. https://doi.org/10.1016/i.ejmech.2017.09.031

24. Saltiel, A. R.; Olefsky, J. M. Diabetes 1996, 45, 1661-1669.

https://doi.org/10.2337/diab.45.12.1661

25. Krische, D. Western J. Med. 2000, 173, 54-57.

26. Ramirez, M. A.; Borja, N. L. Pharmacotherapy 2008, 28, 646-655. https://doi.org/10.1592/phco.28.5.646

27. Zhou, C.; Tang, C.; Chang, E.; Ge, M.; Lin, S.; Cilne, E.; Tan, C. P.; Yue, F.; Zhou, Y. P.; Eiermann, G. J.; Petrov, A.; Salituro, G.; Meinke, P.; Mosley, R.; Akiyama, T. E.; Einstein, M.; Kumar, S.; Berger, J.; Howard, A. D.; Thornberry, N.; Mills S. G.; Yang, L. Bioorg. Med. Chem. Lett. 2010, 20, 1298-1301. https://doi.org/10.1016/i.bmcl.2009.10.052

28. Lugovskoy, A. A.; Degterev, A. I.; Fahmy, A. F.; Zhou, P.; Gross, J. D.; Yuan, J.; Wagner, G. J. Am. Chem. Soc. 2002, 124, 1234-1240.

http://dx.doi.org/10.1021/ja011239y

29. Wadsworth, A.; Moser, M.; Marks, A.; Little, M. S.; Gasparini, N.; Brabec, C. J.; Baran, D.; McCulloch, I. Chem. Soc. Rev. 2019, 48, 1596-1625. http://dx.doi.org/10.1039/C7CS00892A

30. Marinado, T.; Hagberg, D. P.; Hedlund, M.; Edvinsson, T.; Johansson, E. M. J.; Boschloo, G.; Rensmo, H.; Brinck, T.; Sun, L.; Hagfeldt, A. Phys. Chem. Chem. Phys. 2009, 11, 133-141.

http://dx.doi.org/10.1039/B812154K

31. Li, Z.; He, G.; Wan, X.; Liu, Y.; Zhou, J.; Long, G.; Zuo, Y.; Zhang, M.; Chen, Y. Adv. Energy Mater. 2012, 2 , 74-77.

https://doi.org/10.1002/aenm.201100572

32. Meyer, T.; Ogermann, D.; Pankrath, A.; Kleinermanns, K.; Müller, T. J. J. J. Org. Chem. 2012, 77, 37043715. http://dx.doi.org/10.1021/jo202608w

33. Holliday, S.; Ashraf, R. S.; Nielsen, C. B.; Kirkus, M.; Röhr, J. A.; Tan, C.-H.; Collado-Fregoso, E.; Knall, A.-C.; Durrant, J. R.; Nelson, J.; McCulloch, I. J. Am. Chem. Soc. 2015, 137, 898-904.

http://dx.doi.org/10.1021/ja5110602

34. C. E. Redemann, R. N. Icke, G. A. Alles, Org. Synth. 1947, 27, 73-75. http://dx.doi.org/10.15227/orgsyn.027.0073

35. Sucheta; Tahlan S.; Prabhakar, K. V. Chem. Cent. J. 2017, 11, 130-158 https://doi.org/10.1186/s13065-017-0357-2

36. Gabillet, S.; Lecerclé, D.; Loreau, O.; Carboni, M.; Dézard,S.; Gomis, J. M.; Taran, F. Org. Lett. 2007, 9, 3925-3927.

http://dx.doi.org/10.1021/ol701563e

37. Alizadeh, A.; Rostamnia, S.; Zohreh, N.; Hosseinpour, R. Tetrahedron Lett. 2009, 50, 1533-1535. 
https://doi.org/10.1016/i.tetlet.2008.12.107

38. Khodair, A. I. J. Heterocycl. Chem. 2002, 39, 1153-1160.

https://doi.org/10.1002/ihet.5570390607

39. Radi, M.; Botta, L.; Casaluce, G.; Bernardini, M.; Botta, M. J. Comb. Chem. 2010, 12, 200-205. http://dx.doi.org/10.1021/cc9001789

40. Dhara, K.; Paladhi, S.; Midya, G. C.; Dash, J. Org. Biomol. Chem. 2011, 9, 3801-3807. http://dx.doi.org/10.1039/COOB01248C

41. Hassanabadi, A.; Mosslemin, M. H.; Anary-Abbasinejad, M.; Kalantarinejad, A.; Shirazi, M. J. Eur.-J. Chem. 2012, 9, 2074-2078. https://doi.org/10.1155/2012/786024

42. Shah, S.; Singh, B. Bioorg. Med. Chem. Lett. 2012, 22, 5388-5391. https://doi.org/10.1016/i.bmcl.2012.07.049

43. Paladhi, S.; Chauan, A.; Dhara, K.; Tiwari, A. K.; Dash, J. Green Chem. 2012, 14, 2990-2995. http://dx.doi.org/10.1039/C2GC35819K

44. Paladhi, S.; Bhati, M.; Panda, D.; Dash, J. J. Org. Chem. 2014, 79, 1473-1480.

http://dx.doi.org/10.1021/jo402515d

45. Kotha, S.; Sreevani, G. Eur. J. Org. Chem. 2018, 5935-5941. https://doi.org/10.1002/ejoc.201800775

46. Shinde, D. N.; Trivedi, R.; Krishna, J. V. S.; Giribabu, L.; Sridhar, B.; Khursade, P. S.; Prakasham, R. S. New J. Chem. 2018,42, 12587-12594.

http://dx.doi.org/10.1039/C8NJ01598H

47. Harada, K.; Kubo, H.; Abe, J.; Haneta, M.; Conception, A.; Inoue, S.; Okada, S.; Nishioka, K. Bioorg. Med. Chem. 2012, 20, 3242-3254.

https://doi.org/10.1016/i.bmc.2012.03.052

48. Imrich, J.; Bernat, J.; Kristian, P.; Busova, T.; Hocova, S. Collect. Czech. Chem. Commun. 1996, 61, $432-436$. https://doi.org/10.1135/cccc19960432

49. Yang, W.-L.; Tang, F.-F.; He, F.-S.; Li, C.-Y.; Yu, X.; Deng, W.-P. Org. Lett. 2015, 17, 4822-4825. http://dx.doi.org/10.1021/acs.orglett.5b02387

50. Üngören, Ş. H.; Albayrak, S.; Günay, A.;Yurtseven, L.; Yurttas, N. Tetrahedron 2015, 71, 4312-4323. https://doi.org/10.1016/i.tet.2015.04.069

51. Yu, F.; Hu, H.; Gu, X.; Ye, J. Org. Lett. 2012, 14, 2038-2041.

https://doi.org/10.1021/ol300489q

52. Kerdesky, F. A. J.; Holms, J. H.; Moore, J. L.; Bell, R. L.; Dyer, R. D.; Carter, G. W.; Brooks, D. W. J. Med. Chem. 1991, 34, 2158-2165. http://dx.doi.org/10.1021/jm00111a035

53. Wang, T.; Yu, Z.; Hoon, D. L.; Huang, K.-W.; Lan, Y.; Lu, Y. Chem. Sci. 2015, 6, 4912-4922. http://dx.doi.org/10.1039/C5SC01614B

54. Géant, P.-Y.; Urban, M.; Remeš, M.; Císařová, I.; Veselý, J. Eur. J. Org. Chem. 2013, 2013, 7979-7988. https://doi.org/10.1002/ejoc.201300931

55. Wu, W.; Huang, H.; Yuan, X.; Zhu, K.; Ye, J. Chem. Commun. 2012, 48, 9180-9182. http://dx.doi.org/10.1039/C2CC34321E

56. Zhu, K.; Huang, H.; Wu, W.; Wei, Y.; Ye, J. Chem. Commun. 2013, 49, 2157-2159. http://dx.doi.org/10.1039/C3CC00023K

57. Song, Y.-X.; Du, D.-M. J. Org. Chem. 2018, 83, 9278-9290. 
http://dx.doi.org/10.1021/acs.joc.8b01245

58. Zhang, H.; Wang, B.; Cui, L.; Li, Y.; Qu, J.; Song, Y. Org. Biomol. Chem. 2014, 12, 9097-9100. http://dx.doi.org/10.1039/C4OB01921K

59. Kang, T.-C.; Wu, L.-P.; Sha, F.; Wu, X.-Y. Tetrahedron 2018, 74, 1017-1023. https://doi.org/10.1016/i.tet.2017.12.050

60. Xu, H.; Kang, T.-C.; Sha, F.; Wu, X.-Y. Org. Biomol. Chem. 2018,16, 5780-5787. http://dx.doi.org/10.1039/C8OB01524D

61. Huang, Q.; Zhang, L.; Cheng, Y.; Li, P.; Li, W. Adv. Synth. Catal. 2018, 360, 3266-3270. https://doi.org/10.1002/adsc.201800642

62. Diosdado, S.; Etxabe, J.; Izquierdo, J.; Landa, A.; Mielgo, A.; Olaizola, I.; López, R.; Palomo, C. Angew. Chem. Int. Ed. 2013, 52, 11846-11851. http://dx.doi.org/10.1002/anie.201305644

63. Zhu, B.; Qiu, S.; Li, J.; Coote, M. L.; Lee, R.; Jiang, Z. Chem. Sci. 2016, 7, 6060-6067. http://dx.doi.org/10.1039/C6SC02039A

64. Chen, W.; Hartwig, J. F. J. Am. Chem. Soc. 2014, 136, 377-382.

http://dx.doi.org/10.1021/ja410650e

65. Wang, T.-C.; Han, Z.-Y.; Wang, P.-S.; Lin, H.-C.; Luo, S.-W.; Gong, L.-Z. Org. Lett. 2018, 20, 4740-4744. http://dx.doi.org/10.1021/acs.orglett.8b01697

66. Li, J.; Qiu, S.; Ye, X.; Zhu, B.; Liu, H.; Jiang, Z. J. Org. Chem. 2016, 81, 11916-11923. http://dx.doi.org/10.1021/acs.joc.6b02384

67. Zhang, L.; Yuan, H.; Lin, W.; Cheng, Y.; Li, P.; Li, W. Org. Lett. 2018, 20, 4970-4974. http://dx.doi.org/10.1021/acs.orglett.8b02088

\section{Authors' Biographies}

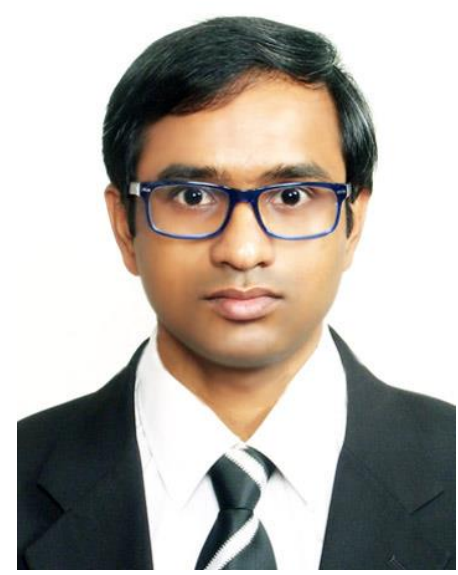

Dr. Sushovan Paladhi was born in West Bengal, India. He completed his B.Sc. and M.Sc. degree in chemistry from Vidyasagar University, West Bengal and obtained his Ph.D. in organic chemistry under the supervision of Prof. Jyotirmayee Dash from IISER-Kolkata, India in 2014. Later, he worked as a project fellow at IACS for 6 months and then moved to Sungkyunkwan University, South Korea in 2015 as a post-doctoral fellow in the group of Prof. Choong Eui Song, where he worked until August 2017. He then moved to India and joined in November 2017 as an assistant professor in the postgraduate department of chemistry, Anugrah Narayan Singh College, Barh, Patna (a constituent unit of Patliputra University, Patna, India). Currently, he is the head 
of the chemistry department. During his research career, he was selected for several awards and fellowships like "2014 Lily outstanding Thesis Award", "SERB-Overseas Postdoctoral Fellowship 2015-16", "BK21 Postdoctoral Fellowship 2016-2017" etc. His research interests include the development of new synthetic protocol under environmentally benign reaction conditions.

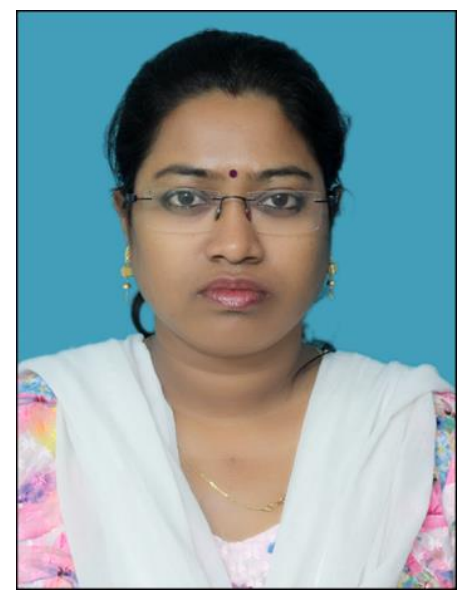

Dr. Barnali Jana was born in West Bengal, India. She completed her B.Sc. and M.Sc. degree in chemistry from Vidyasagar University, West Bengal with gold medals and obtained her Ph.D. in physical chemistry under the supervision of Prof. Nitin Chattopadhyay from Jadavpur University, West Bengal, India in 2014. Since 2015, she is working as an assistant professor in the postgraduate department of chemistry, Haldia Government College, West Bengal, India (under the affiliation of Vidyasagar University, West Bengal, India) Her research interest is the synthesis of new molecules for photophysical applications.

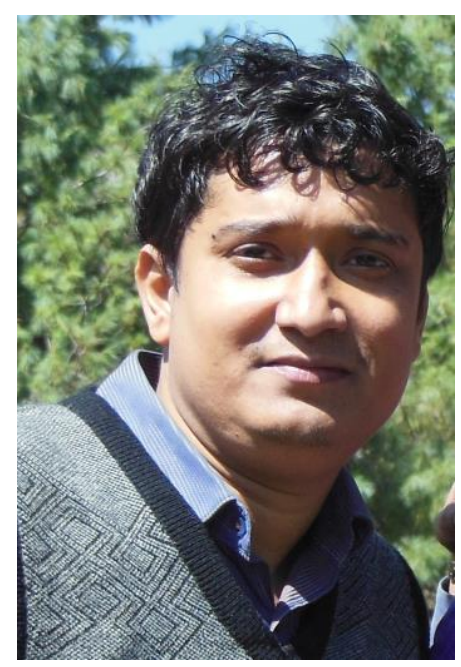

Dr. Sudipta Pathak is currently working as an assistant professor in the postgraduate department of chemistry, Haldia Government College, Debhog, West Bengal, India. He completed his B.Sc. degree in chemistry from Vidyasagar University, West Bengal and his M.Sc. degree from IIT Bombay, Mumbai, India. He received his Ph.D. degree in 2015 from Calcutta University, Kolkata, India. His research interest include the synthesis of new organic molecules. 


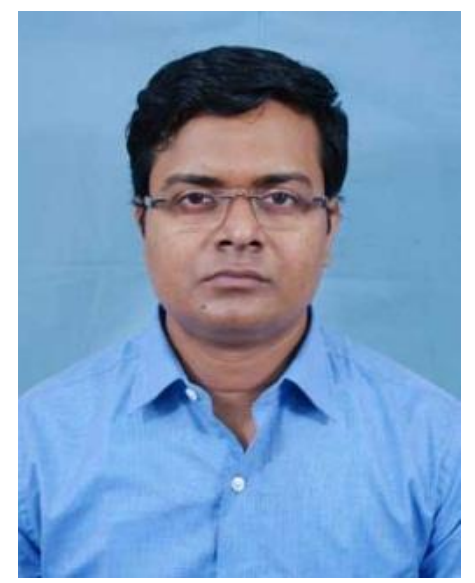

Dr. Saikat Kumar Manna is currently working as an assistant professor in the postgraduate department of chemistry, Haldia Government College, Debhog, West Bengal, India. He completed his B.Sc. and M.Sc. degrees in chemistry from Vidyasagar University, West Bengal and received his Ph.D. degree from Indian Institute of Engineering Science and Technology, Shibpur, Howrah, India in 2015. His research interest include the development of new organic chemosensors for the detection of transition metals. 